\title{
Problèmes Mixtes pour l'Équation des Ondes II
}

\author{
Par \\ Mitsuru IKAWA
}

\section{$\S 1$. Introduction}

Soit $\mathcal{O}$ un objet borné dans $\boldsymbol{R}^{3}=\left\{x ; x=\left(x_{1}, x_{2}, x_{3}\right), x_{j} \in \boldsymbol{R}\right\}$ dont la frontière $\Gamma$ est une surface indéfiniment différentiable, fermée et simple. Posons $\Omega=\boldsymbol{R}^{3}-\mathcal{O}-\Gamma$.

Soit

$$
B=\sum_{j=1}^{3} b_{j}(x) \frac{\partial}{\partial x_{j}}+c(x) \frac{\partial}{\partial t}+d(x)
$$

un opérateur différentiel du premier order à coefficients indéfiniment différentiables définis dans un voisinage de $\Gamma$. Supposons que

$$
b_{j}(x), j=1,2,3 \text { et } c(x) \text { sont à valeurs réelles }
$$

$$
\sum_{j=1}^{3} b_{j}(x) n_{j}(x)=1 \quad \text { sur } \quad \Gamma
$$

où $n(x)=\left(n_{1}(x), n_{2}(x), n_{3}(x)\right)$ désigne la normale unitaire extérieure par rapport à $\mathcal{O}$ de $\Gamma$ en $x$.

Considérons le problème mixte

$$
\left\{\begin{array}{l}
\square u(x, t)=\frac{\partial^{2} u}{\partial t^{2}}-\sum_{j=1}^{3} \frac{\partial^{2} u}{\partial x_{j}^{2}}=f(x, t) \quad \text { dans } \quad \Omega \times(0, \infty) \\
B u(x, t)=g(x, t) \quad \text { sur } \quad \Gamma \times(0, \infty) \\
u(x, 0)=u_{0}(x) \\
\frac{\partial u}{\partial t}(x, 0)=u_{1}(x) .
\end{array}\right.
$$

On dit que le problème mixte $(P)$ représente un phénomène propagateur avec une vitesse finie lorsqu'il existe une constante $\gamma>0$ avec la propriété suivante:

Communiqué par S. Matsuura, le 4 mai, 1976.

* Département de Mathématiques, Université d'Osaka, Toyonaka 560, Japan. 
pour $\left(x_{0}, t_{0}\right) \in \bar{\Omega} \times[0, \infty)$ et $u(x, t) \in C^{\infty}(\bar{\Omega} \times[0, \infty))$ quelconques les égalités

$$
\begin{array}{ll}
\square u=0 \quad \text { dans } & \Omega \times[0, \infty) \cap \Lambda\left(x_{0}, t_{0}\right) \\
B u=0 \quad \text { sur } \quad \Gamma \times[0, \infty) \cap \Lambda\left(x_{0}, t_{0}\right) & \\
u(x, 0)=\frac{\partial u}{\partial t}(x, 0)=0 \quad \text { dans } \bar{\Omega} \times\{t=0\} \cap \Lambda\left(x_{0}, t_{0}\right)
\end{array}
$$

entraîne $u\left(x_{0}, t_{0}\right)=0$, où

$$
\Lambda\left(x_{0}, t_{0}\right)=\left\{(x, t) ;\left|x-x_{0}\right| \leq \gamma\left(t_{0}-t\right)\right\} .
$$

Et puis dans cet article l'inférieur des $\gamma$ avec la propriété au-dessus est dit la vitesse maximume du problème mixte $(\mathrm{P})$.

Le fait que nous allons montrer dans cet article est le suivant:

Théorème. Supposons que la courbure Gaussienne de $\Gamma$ soit strictement positive. Pour que $(\mathrm{P})$ soit bien posé au sens de $C^{\infty}$ et ait une vitesse finie de propagation il faut et il suffit, sous les restrictions (1. 1) et (1.2), qu'il ait lieu

$$
c(x)<1 \quad \text { pour tout } \quad x \in \Gamma .
$$

Et lorsque (1.3) a lieu la vitesse maximume de propagation du problème mixte $(\mathrm{P})$ est égale à

$$
\max \left(1, \sup _{x \in \Gamma}-\frac{1+v(x)^{2}}{-c(x) v(x)+\sqrt{1+\left|v(x)^{2}-c(x)^{2}\right|}}\right)
$$

où

$$
v(x)=\left(\sum_{j=1}^{3}\left(b_{j}(x)-n_{j}(x)\right)^{2}\right)^{1 / 2}
$$

Dans l'article précédent nous avons considéré les problèmes mixtes dans un domaine de $\boldsymbol{R}^{2}$ qui est l'extérieur d'un objet convexe. Comme nous l'avons remarqué la supposition sur le domaine joue un rôle très important. Nous voulons souligner encore que le problème $(\mathrm{P})$ n'est plus bien posé au sens de $C^{\infty}$ en général sous les condition (1.1), (1.2) et (1.3) au cas où le domaine n'est pas l'extérieur d'un objet convexe. A propos de ce remarque, nous avons considéré dans [3] les propriétés 
fondamentales des problèmes mixtes pour l'équation des ondes dans un domaine de $\boldsymbol{R}^{3}$ qui est l'intérieur d'une surface fermée.

\section{$\S$ 2. Réduction du Problème}

Soit $\varphi(x)$ une fonction à valeurs réelles vérifiant $\nabla \varphi \in \mathscr{B}\left(\mathbb{R}^{3}\right)$ et $\sup _{x \in \boldsymbol{R}^{3}} \mid \nabla \varphi(x):<1$.

Posons

$$
\begin{aligned}
& A_{\varphi}(\partial / \partial t, \partial / \partial x)=\left(1-(\nabla \varphi)^{2}\right) \frac{\partial^{2}}{\partial t^{2}}-2 \nabla \varphi \cdot \nabla \frac{\partial}{\partial t}-\Delta-\Delta \varphi \frac{\partial}{\partial t} \\
& B_{\varphi}(\partial / \partial t, \partial / \partial x)=\sum_{j=1}^{3} b_{j}(x)\left(\frac{\partial}{\partial x_{j}}+\frac{\partial \varphi}{\partial x_{j}} \frac{\partial}{\partial t}\right)+c(x) \frac{\partial}{\partial t}+d(x)
\end{aligned}
$$

et considérons le problème mixte

$\left(\mathrm{P}_{\varphi}\right) \quad\left\{\begin{array}{lll}A_{\varphi} u=f(x, t) & \text { dans } & \Omega \times(0, \infty) \\ B_{\varphi} u=g(x, t) & \text { sur } & \Gamma \times(0, \infty) \\ u(x, 0)=u_{0}(x) & \\ \frac{\partial u}{\partial t}(x, 0)=u_{1}(x) . & \end{array}\right.$

Afin de montrer la résolubilité du problème $\left(\mathrm{P}_{\varphi}\right)$ nous considérons le problème au bord avec un paramètre $p=i k+\mu, \mu>0$

$$
\begin{array}{lll}
A_{\varphi}(p, \partial / \partial x) w(x)=0 & \text { dans } \quad \Omega \\
B_{\varphi}(p, \partial / \partial x) w(x)=h(x) & \text { sur } \quad \Gamma .
\end{array}
$$

L'important est d'obtenir les estimations à priori des solutions de (2.1) pour tout $p$ à partie imaginaire suffisamment grande.

Noter que le problème au bord

$$
\begin{cases}A_{\varphi}(p, \partial / \partial x) u(x)=0 & \text { dans } \Omega \\ u(x)=g(x) & \text { sur } \Gamma\end{cases}
$$

admet une solution unique dans $\bigcap_{m \geq 0} H^{m}(\Omega)$ pour toute $g(x) \in C^{\infty}(\Gamma)$ et qu'il a lieu l'estimation

$$
\sum_{l+l^{\prime} \leq m}|p|^{l}\|w(x)\|_{l^{\prime}} \leq C_{m, \varphi}\|g\|_{m+1}
$$


lorsque $\operatorname{Re} p=\mu \geq \mu_{\varphi}, \quad \mu_{\varphi}$ une certaine constante. ${ }^{1)}$

Désignons par $U_{\varphi}(p, g: x)$ la solution $u(x)$ de (2.2) pour la donnée $g(x) \in C^{\infty}(\Gamma)$ et définissons $\mathscr{B}_{\varphi}(p)$ par

$$
\mathscr{B}_{\varphi}(p) g=\left.B_{\varphi}(p, \partial / \partial x) U_{\varphi}(p, g: x)\right|_{\Gamma} .
$$

Alors $\mathscr{B}_{\varphi}(p)$ est une application linéaire de $C^{\infty}(\Gamma)$ dans $C^{\infty}(\Gamma)$.

Concernant l'opérateur $\mathscr{B}_{\varphi}(p)$ admettons le

Théorème 2.1. Nous nous plaçons dans l'hypothèse sur $\Omega$ faite dans le théorème de l'introduction. Alors pour $\operatorname{Re} p \geq \mu_{\varphi}$ on a

$$
-\operatorname{Re}\left(\mathscr{B}_{\varphi}(p) g, g\right)_{m} \geq\left(\mu c_{0}(\varphi)-C\right)\|g\|_{m}^{2}-C_{\varphi, m}\|g\|_{0}^{2}
$$

pour toute $g \in C^{\infty}\left(I^{\prime}\right)$, où

$$
\begin{aligned}
& c_{0}(\varphi)=\inf _{x \in \Gamma}\left(\sqrt{1-\left|\varphi_{s}\right|^{2}}-v\left|\varphi_{s}\right|-c\right) \\
& \varphi_{s}=\nabla \varphi-(\nabla \varphi \cdot n) n
\end{aligned}
$$

et $C_{\varphi, m}$ est une constante indépendante de $p$ et de $g$.

$$
\text { Posons } b_{j}^{\prime}(x)=2 n_{j}(x)-b_{j}(x), d^{\prime}(x)=\overline{d(x)}+\sum_{j=1}^{3} \frac{\partial}{\partial x_{j}}\left(n_{j}(x)-b_{j}(x)\right)
$$
et puis

$$
B^{\prime}=\sum_{j=1}^{3} b_{j}^{\prime}(x) \frac{\partial}{\partial x_{j}}+c(x) \frac{\partial}{\partial t}+d^{\prime}(x)
$$

Alors il a lieu

$$
\begin{aligned}
& \left(\left(A_{\varphi}(p, \partial / \partial x) V, W\right)\right)_{0}-\left\langle\left(V, A_{-\varphi}(\bar{p}, \partial / \partial x) W\right)\right)_{0} \\
& \quad=\left(B_{\varphi}(p, \partial / \partial x) V, W\right)_{0}-\left(V, B_{-\varphi}^{\prime}(\bar{p}, \partial / \partial x) W\right)_{0}
\end{aligned}
$$

pour toutes $V, W \in C^{\infty}(\bar{\Omega}) \cap H^{2}(\Omega)$. Donc si l'on définit $\mathscr{B}_{\varphi}^{\prime}(p)$ par

$$
\mathscr{B}_{\varphi}^{\prime}(p) g=\left.\mathscr{B}_{\varphi}^{\prime}(p, \partial / \partial x) U_{\varphi}(p, g: x)\right|_{\Gamma},
$$

on déduit de (2.4)

$$
\left(\mathscr{B}_{\varphi}(p) g, h\right)_{0}=\left(g, \mathscr{B}_{-\varphi}^{\prime}(\bar{p}) h\right)_{0}, \forall g, h \in C^{\infty}(\Gamma) .
$$

D'après le théorème 2.1 les relations

1) Voir, par exemple, Sakamoto [11]. " $\mid \|_{\mid m m}$ et $\|^{\cdot}{ }_{\mid m}$ désignent les normes des espaces de Sobolev $H^{m}(\Omega)$ et $H^{m}(\Gamma)$ respectivement. 


$$
\begin{aligned}
& \sum_{j=1}^{3} b_{j}^{\prime}(x) n_{j}(x)=1 \\
& \sum_{j=1}^{3}\left(b_{j}^{\prime}(x)-n_{j}(x)\right)^{2}=\sum_{j=1}^{3}\left(b_{j}(x)-n_{j}(x)\right)^{2}
\end{aligned}
$$

résultent qu'il a lieu l'estimation

$$
-\operatorname{Re}\left(\mathscr{B}_{-\varphi}^{\prime}(p) h, h\right)_{m} \geq\left(\mu c_{0}(\varphi)-C\right)\|h\|_{m}^{2}-C_{\varphi, m}^{\prime}\|h\|_{0}^{2} .
$$

L'inégalité

$$
\sup _{x \in \Gamma}\left|\varphi_{s}\right|<\inf _{x \in \Gamma}\left(-c(x) v(x)+\sqrt{1+\left|v(x)^{2}-c(x)^{2}\right|}\right)\left(1+v(x)^{2}\right)^{-1}
$$

entraîne $c_{0}(\varphi)>0$. Par conséquent, on peut démontrer selon le raisonnement analogue à celui fait dans $\$ 3$ de [4] que, lorsque $\varphi(x)$ vérifie (2.5), le problème (2.1) admet une solution unique $w(x)$ dans $\bigcap_{m \geq 0} H^{m}(\Omega)$ pour $h(x) \in C^{\infty}(\Gamma)$ si $\operatorname{Re} p \geq \tilde{\mu}_{\varphi}$, où $\tilde{\mu}_{\varphi}$ est une certaine constante. Désignons par $F(p, h: x)$ la solution de (2.1) pour la donnée $h(x)$ $\in C^{\infty}(\Gamma)$. Alors on a

$$
\sum_{l+l^{\prime} \leq m}|p|^{l}\|F(p, h: x)\|_{l^{\prime}} \leq C_{m, \varphi}\|h\|_{m+1} .
$$

Alors on déduit immédiat des faits au-dessus que le problème

$$
\left\{\begin{array}{l}
A_{\varphi}(\partial / \partial t, \partial / \partial x) w(x, t)=0 \quad \text { dans } \quad \Omega \times(-\infty, \infty) \\
B_{\varphi}(\partial / \partial t, \partial / \partial x) w(x, t)=h(x, t) \quad \text { sur } \Gamma \times(-\infty, \infty)
\end{array}\right.
$$

admet une solution unique $w(x, t)$ dans $\left.H_{\mu}^{m}(\Omega) \times(-\infty, \infty)\right)$ pour la donnée au bord $h(x, t) \in H_{\mu}^{m+1}(\Gamma \times(-\infty, \infty))$ si $\mu \geq \widetilde{\mu}_{\varphi}, m \geq 2$. Et plus on peut montrer que

$$
\operatorname{supp} h(x, t) \subset \Gamma \times\left[t_{0}, \infty\right)
$$

entraîne

$$
\operatorname{supp} w(x, t) \subset \Omega \times\left[t_{0}, \infty\right)
$$

Donc on a le

Théorème 2. 2. Soit $\varphi(x)$ une fonction à valeurs réelles vérifiant $\nabla \varphi \in \mathscr{B}\left(\boldsymbol{R}^{3}\right), \sup _{x \in \boldsymbol{R}^{3}}|\nabla \varphi(x)|<1$ el (2.5). Pour $u_{0}, u_{1} \in H^{m+2}(\Omega), f \in H_{\mu}^{m+2}$ $(\Omega \times(0, \infty)), g \in H_{\mu}^{m+2}(\Gamma \times(0, \infty))$ le problème mixte $\left(\mathrm{P}_{\varphi}\right)$ admet une solution unique $u(x, t)$ dans $H_{\mu}^{m}(\Omega \times(0, \infty))$ si $u_{0}, u_{1}, f$ et $g$ satisfont à 
la condition de compatibilité d'ordre $m \geq 2$ et si $\mu \geq \widetilde{\mu}_{\varphi}$. Lorsque $u_{0}=u_{1}$ $=0$ et $f(x, t)=0, g(x, t)=0$ pour $0 \leq t \leq t_{0}$ on a $u(x, t)=0$ pour $0 \leq t \leq t_{0}$.

Supposons que (1.3) ait lieu. Le théorème 2.2 montre que le problème $(\mathrm{P})$ est bien posé au sens de $C^{\infty}$ puisque une fonction $\varphi(x) \equiv 0$ satisfait à (2.5). Démontrons que la vitesse propagatrice du problème (P) est au plus (1.4). D'abord considérons l'unicité locale des solutions de $\left(\mathrm{P}_{\varphi}\right)$ pour $\varphi$ vérifiant (2.5).

Soit $x_{0} \in \Gamma$. Supposons que $u(x, t) \in C^{\infty}(\bar{\Omega} \times[0, \infty))$ est une solution de $\left(\mathrm{P}_{\varphi}\right)$ et que pour un voisinage $U$ de $x_{0}$ dans $\mathbb{R}^{3}$ et $t_{0}>0$

$$
\begin{array}{lll}
u_{0}(x)=u_{1}(x)=0 & \text { dans } & \bar{\Omega} \cap \mathcal{U} \\
f(x, t)=0 & \text { dans } & (\bar{\Omega} \cap \mathcal{U}) \times\left[0, t_{0}\right] \\
g(x, t)=0 & \text { sur } & (\Gamma \cap \mathcal{U}) \times\left[0, t_{0}\right] .
\end{array}
$$

Prenons une fonction $\psi(x)$ de telle façon que

$$
\begin{array}{lll}
\psi(x)=\left|x-x_{0}\right|^{2} & \text { dans } & U \\
\psi(x) \geq c>0 & \text { dans } & C U
\end{array}
$$

et que $\varphi+\psi$ satisfasse à (2.5). C'est bien possible en prenant $U$ suffisamment petit. Après le changement des variables

$$
\left\{\begin{array}{l}
t^{\prime}=t+\psi(x) \\
x^{\prime}=x
\end{array}\right.
$$

la fonction $u\left(x^{\prime}, t^{\prime}\right)$ définie par

$$
\widetilde{u}\left(x^{\prime}, t^{\prime}\right)=\widetilde{u}(x, t+\psi(x))=u(x, t) \quad \text { pour } \quad(x, t) \in(\bar{\Omega} \cap U) \times\left[0, t_{0}\right]
$$

et $\widetilde{u}\left(x^{\prime}, t^{\prime}\right)=0$ pour $\left(x^{\prime}, t^{\prime}\right)$ tel que $t^{\prime} \leq \phi\left(x^{\prime}\right)$. Alors on voit que $\widetilde{u}\left(x^{\prime}, t^{\prime}\right) \in C^{2}\left(\bar{\Omega} \times\left[0, t_{0}\right]\right)$ et qu'elle vérifie

$$
\begin{array}{lll}
A_{\varphi+\psi}\left(\partial / \partial t^{\prime}, \partial / \partial x^{\prime}\right) \widetilde{u}=0 & \text { pour } & t^{\prime} \leq t_{0} \\
B_{\varphi+\psi}\left(\partial / \partial t^{\prime}, \partial / \partial x^{\prime}\right) \widetilde{u}=0 & \text { pour } & t^{\prime} \leq t_{0} \\
\widetilde{u}\left(x^{\prime}, 0\right)=\frac{\partial \widetilde{u}}{\partial t^{\prime}}\left(x^{\prime}, 0\right)=0 . &
\end{array}
$$

Puisque $\varphi+\psi$ satisfait à la condition du théorème 2.2 il se résulte que $\widetilde{u}\left(x^{\prime}, t^{\prime}\right)$ s'annule pour $t^{\prime} \leq t_{0}$, d'où on déduit que $u(x, t)=0$ dans 
$\left(\bar{\Omega} \cap U^{\prime}\right) \times\left[0, t_{0}^{\prime}\right]$ pour un voisinage $U^{\prime}$ de l'origine et $t_{0}^{\prime}>0$. Il est évident que l'on a l'unicité locale des solutions dans un voisinage de $x_{0} \notin \Gamma$. Donc on a la

Proposition 2.3. A propos du problème mixte $\left(\mathrm{P}_{\varphi}\right)$, si $\varphi$ satisfait à (2.5) il a lieu l'unicité locale des solutions.

Nous allons considérer la vitesse propagatrice de (P). Posons

$$
v_{0}=\max \left(1, \sup _{x \in \Gamma}\left(1+v^{2}\right)\left(-c v+\sqrt{1+\left|v^{2}-c^{2}\right|}\right)^{-1}\right)
$$

et

$$
\Lambda\left(x_{0}, t_{0}\right)=\left\{(x, t) ; x \in \bar{\Omega},\left|x-x_{0}\right| \leq v_{0}\left(t_{0}-t\right)\right\}
$$

pour $\left(x_{0}, t_{0}\right) \in \bar{\Omega} \times[0, \infty)$.

Supposons que $u(x, t) \in C^{\infty}(\bar{\Omega} \times(-\infty, \infty))$ vérifie

$$
\left\{\begin{array}{lll}
\square u=0 & \text { dans } & \bar{\Omega} \times(-\infty, \infty) \cap \Lambda\left(x_{0}, t_{0}\right) \\
B u=0 & \text { sur } & \Gamma \times(-\infty, \infty) \cap \Lambda\left(x_{0}, t_{0}\right) \\
u(x, t)=0 & \text { dans } & \Lambda\left(x_{0}, t_{0}\right) \cap\{t \leq 0\} .
\end{array}\right.
$$

Posons

$$
\begin{gathered}
\varphi_{r}(x)=\sqrt{\gamma+\frac{1}{v_{0}^{2}}\left|x-x_{0}\right|^{2}}-t_{0} \\
\Lambda_{r}\left(x_{0}, t_{0}\right)=\left\{\left(x^{\prime}, t^{\prime}\right) ; t^{\prime}=t+\varphi_{r}(x), x^{\prime}=x,(x, t) \in \Lambda\left(x_{0}, t_{0}\right)\right\} \\
G_{r}\left(x_{0}, t_{0}\right)=\Lambda_{r}\left(x_{0}, t_{0}\right) \cap(\Gamma \times(-\infty, \infty))
\end{gathered}
$$

et

$$
\Omega_{r}=\left\{x \in \Omega, \varphi_{r}(x) \leq 0\right\}
$$

pour $0 \leq r \leq t_{0}^{2}$.

Par le changement des variables

$$
\left\{\begin{array}{l}
x^{\prime}=x \\
t^{\prime}=t+\varphi_{r}(x),
\end{array}\right.
$$

(2.7) se transforme à

$$
\left\{\begin{array}{lll}
A_{\varphi_{r}} u_{r}\left(x^{\prime}, t^{\prime}\right)=0 & \text { dans } & \Lambda_{r}\left(x_{0}, t_{0}\right) \\
B_{\varphi_{r}} u_{r}\left(x^{\prime}, t^{\prime}\right)=0 & \text { sur } & G_{r}\left(x_{0}, t_{0}\right),
\end{array}\right.
$$


où $u_{r}\left(x^{\prime}, t^{\prime}\right)=u_{r}\left(x, t+\varphi_{r}(x)\right)=u(x, t)$.

Supposons que $u_{r}\left(x^{\prime}, 0\right)=0$ dans $\bar{\Omega}_{r}$ pour $0<a \leq \gamma \leq t_{0}^{2}$. Alors nous avons

$$
\left\{\begin{array}{lll}
A_{\varphi_{a}} u_{a}\left(x^{\prime}, t^{\prime}\right)=0 & \text { dans } & \Lambda_{a}\left(x_{0}, t_{0}\right) \\
B_{\varphi_{a}} u_{a}\left(x^{\prime}, t^{\prime}\right)=0 & \text { sur } & G_{a}\left(x_{0}, t_{0}\right) \\
u_{a}\left(x^{\prime}, 0\right)=\frac{\partial u_{a}}{\partial t^{\prime}}\left(x^{\prime}, 0\right)=0 & \text { dans } \quad \bar{\Omega}_{a} .
\end{array}\right.
$$

En tenant compte que $\varphi_{r}(x)$ satisfait à $(2.5)$ si $\gamma>0$ l'application de la proposition 2.3 à $(2.8)$ déduit que $\widetilde{u}_{a}\left(x^{\prime}, t^{\prime}\right)=0$ dans $\overline{\Omega_{a-\varepsilon}} \times[0, \varepsilon]$, où $\varepsilon$ est une constante positive. Cela signifie que $u_{r}\left(x^{\prime}, 0\right)=0$ pour tout $a-\varepsilon \leq r \leq t_{0}^{2}$. De cette façon on montre que $u_{r}\left(x^{\prime}, 0\right)=0$ pour tout $0<r \leq t_{0}^{2}$, à savoir, $u(x, t)=0$ dans $\Lambda\left(x_{0}, t_{0}\right)$.

Donc nous avons démontré que la vitesse maximume $d u$ problème mixte (P) est majorée par (1.4).

D'autre part, on peut montrer que la vitesse maximume est au moins (1.4). Ça sera démontré dans $\S 9$, où nous allons démontrer aussi qu'il faut la condition (1.3) pour que (P) soit bien posé au sens de $C^{\infty}$ et que représente un phénomène avec une vitesse finie.

Dans les paragraphes 3-8 nous démontrons le théorème 2.1.

\section{$\S$ 3. Décomposition des Fonctions sur $\Gamma$}

Nous allons construire une fonction $u(x)$ vérifiant (2.2) asymptotiquement par rapport à $|p|$.

$s$ désigne un point sur $\Gamma$. Supposons que l'origine 0 appartient à $\Gamma$ et que $n(0)=(0,0,1)$. Nous commençons la construction de $u(x)$ par une décomposition de $g(x) \in C^{\infty}(\Gamma)$ dont le support est contenu dans un voisinage suffisamment petit de l'origine.

Supposons que $\Gamma$ est représentée dans un voisinage $\Gamma_{0}$ de l'origine comme

$$
s(\sigma)=\left(s_{1}\left(\sigma_{1}, \sigma_{2}\right), s_{2}\left(\sigma_{1}, \sigma_{2}\right), s_{3}\left(\sigma_{1}, \sigma_{2}\right)\right)
$$

par les paramètres $\sigma=\left(\sigma_{1}, \sigma_{2}\right) \in I_{\sigma}=\left[-\sigma_{10}, \sigma_{10}\right] \times\left[-\sigma_{20}, \sigma_{20}\right]$ de telle façon que 


$$
\begin{aligned}
& \frac{\partial s}{\partial \sigma_{1}} \cdot \frac{\partial s}{\partial \sigma_{2}}=0 \quad \forall \sigma \in I_{\sigma} \\
& s(0)=0 \\
& \frac{\partial s}{\partial \sigma_{1}}(0)=(1,0,0), \quad \frac{\partial s}{\partial \sigma_{2}}(0)=(0,1,0) .
\end{aligned}
$$

Désignons $\left(\frac{\partial \varphi(s)}{\partial \sigma_{1}}, \frac{\partial \varphi(s)}{\partial \sigma_{2}}\right)_{\sigma=0} \operatorname{par} \varphi^{0}$. Prenons des fonctions à valeurs réelles $\chi_{j}(l) \in C^{\infty}\left(\boldsymbol{R}^{1}\right), j=1,2,3$ de façon que

$$
\begin{aligned}
& \chi_{1}(l)= \begin{cases}1 & l<1-2 \alpha_{0} \\
0 & l>1-\alpha_{0}\end{cases} \\
& \chi_{2}(l)= \begin{cases}1 & |l-1| \leq \alpha_{0} \\
0 & |l-1| \geq 2 \alpha_{0}\end{cases} \\
& \chi_{3}(l)= \begin{cases}1 & l>1+2 \alpha_{0} \\
0 & l<1+\alpha_{0}\end{cases}
\end{aligned}
$$

et que

$$
\sum_{j=1}^{3} \chi_{j}(l)^{2}=1 \quad \text { pour tout } \quad l \in \mathbb{R}
$$

où $\alpha_{0}$ est une constante positive qui sera déterminée suffisamment petite plus tard. Soit $\Gamma_{1}$ un voisinage de l'origine tel que $\bar{\Gamma}_{1} \subset \Gamma_{0}$. Alors pour toute $g(s) \in \mathscr{D}\left(\Gamma_{1}\right)$ on a

$$
g(s(\sigma))=\int_{\boldsymbol{R}^{2}} d \xi \int_{I_{\sigma}} d \sigma^{\prime} \exp \left\{i\left\langle\sigma-\sigma^{\prime}, \xi\right\rangle\right\} g\left(s\left(\sigma^{\prime}\right)\right) .
$$

Définissons $V_{j} g, j=1,2,3$ par

$$
V_{j} g(s(\sigma))=\lambda(s(\sigma)) \int_{\boldsymbol{R}^{2}} d \xi \int_{I_{\sigma}} d \sigma^{\prime} \exp \left\{i\left\langle\sigma-\sigma^{\prime}, \xi\right\rangle\right\} \chi_{j}\left(\xi / k+\varphi^{0}\right)^{2} g\left(s\left(\sigma^{\prime}\right)\right)
$$

où $\lambda(s) \in \mathscr{D}\left(\Gamma_{0}\right)$ vérifiant $\lambda(s)=1$ sur $\Gamma_{1}$. Alors il est évident que

$$
\sum_{j=1}^{3} V_{j} g(s)=g(s) \quad \text { pour toute } g \in \mathscr{D}\left(\Gamma_{1}\right) .
$$

En posant $\xi=k\left\{(1+\alpha) \xi^{\prime}-\varphi^{0}\right\}, \alpha \geq-1, \xi^{\prime} \in \Sigma=\left\{\left(\xi_{1}, \xi_{2}\right) ; \xi_{1}^{2}+\xi_{2}^{2}=1\right\}$, on a (3.2) $V_{j} g(s(\sigma))=\lambda(s(\sigma)) \int_{\Sigma} d \xi^{\prime} \int_{-1}^{\infty} d \alpha \int_{I_{\sigma}} d \sigma^{\prime} \exp \left[i k\left\{(1+\alpha)\left\langle\sigma-\sigma^{\prime}, \xi^{\prime}\right\rangle\right.\right.$ 


$$
\left.\left.-\left\langle\sigma-\sigma^{\prime}, \varphi^{0}\right\rangle\right\}\right] \chi_{j}(1+\alpha)^{2} k^{2}(1+\alpha) g\left(s\left(\sigma^{\prime}\right)\right) .
$$

D'après la définition de $\chi_{2}(l)$

$$
\begin{aligned}
V_{2} g(s(\sigma))= & \lambda(s(\sigma)) \int_{\Sigma} d \xi^{\prime} \int_{I_{\alpha}} d \alpha \int_{I_{\sigma}} d \sigma^{\prime} \exp \left[i k \left\{(1+\alpha)\left\langle\sigma-\sigma^{\prime}, \xi^{\prime}\right\rangle\right.\right. \\
& \left.\left.-\left\langle\sigma-\sigma^{\prime}, \varphi^{0}\right\rangle\right\}\right] \chi_{2}(1+\alpha)^{2} k^{2}(1+\alpha) g\left(s\left(\sigma^{\prime}\right)\right)
\end{aligned}
$$

où $I_{\alpha}=\left[-2 \alpha_{0}, 2 \alpha_{0}\right]$.

Nous construisons $U_{j}(p, g: x), j=1,2,3$, des fonctions satisfaisant asymptotiquement à

$$
\left\{\begin{array}{l}
A_{\varphi} U_{j}(p, g: x)=0 \quad \text { dans } \Omega \\
U_{j}(p, g: x)=V_{j} g \quad \text { sur } \Gamma .
\end{array}\right.
$$

La construction de $U_{2}$ est plus difficile que les autres. Nous commençons à construire $U_{2}$ par l'introduction $\theta_{0}(x, \eta), \rho_{0}(x, \eta)$ des fonctions définies dans $\bar{\Omega}$ avec un paramètre $\eta \in \Sigma$. Pour $\eta \in \Sigma$ posons

$$
\mathscr{C}_{\eta}=\left\{\sigma ;\left(\eta_{1} \sigma_{1}+\eta_{2} \sigma_{2}\right)=-\left(-\eta_{2} \sigma_{1}+\eta_{1} \sigma_{2}\right)^{2}\right\}
$$

et prenons une fonction $\tilde{f}(\sigma, \eta)$ définie dans un voisinage de $\sigma=0$ de telle façon que

$$
\begin{aligned}
& \tilde{f}(\sigma, \eta)=0 \quad \text { sur } \mathscr{C}_{\eta} \\
& \left.\left(\frac{\partial \tilde{f}}{\partial \sigma_{1}}, \frac{\partial \tilde{f}}{\partial \sigma_{2}}\right)\right|_{\sigma=0}=\eta
\end{aligned}
$$

et

$$
\left(\frac{\partial s}{\partial \sigma_{1}}\right)^{-2}\left(\frac{\partial \tilde{f}}{\partial \sigma_{1}}\right)^{2}+\left(\frac{\partial s}{\partial \sigma_{2}}\right)^{-2}\left(\frac{\partial \tilde{f}}{\partial \sigma_{2}}\right)^{2}=1
$$

Le théorème de Hamilton-Jacobi assure l'existence unique de telle fonction $\tilde{f}$. En choisissant $\Gamma_{0}$ suffisamment petit, une fonction $f(s, \eta)$ définie par $f(s(\sigma), \eta)=\tilde{f}(\sigma, \eta)$ appartient à $C^{\infty}\left(\Gamma_{0}\right)$ pour tout $\eta \in \Sigma$ et satisfait à

$$
(\nabla f(s, \eta))^{2}=1 \quad \text { dans } \quad \Gamma_{0}
$$

et à

$$
(\nabla f)(0, \eta)=\eta_{1} \frac{\partial}{\partial \sigma_{1}}+\eta_{2} \frac{\partial}{\partial \sigma_{2}}
$$

Puisque la courbure Gaussienne de $\Gamma$ est strictement positive, en suivant 
le raisonnement de Ludwig [7], on peut construire des fonctions $\theta_{0}(x, \eta)$, $\rho_{0}(x, \eta) \in C^{\infty}(U \times \Sigma)$, où $U$ est un voisinage de l'origine dans $\mathbb{R}^{3}$, avec les propriétés suivantes:

$$
\left\{\begin{array}{lll}
\left(\nabla \theta_{0}\right)^{2}+\rho_{0}\left(\nabla \rho_{0}\right)^{2}=1 & \text { dans } & \bar{\Omega} \cap \mathcal{U} \\
\nabla \theta_{0} \cdot \nabla \rho_{0}=0 & \text { dans } \bar{\Omega} \cap \mathcal{U} \\
\theta_{0}(s, \eta)=f(s, \eta) & \text { sur } \quad \Gamma \cap \mathcal{} .
\end{array}\right.
$$

Remarquons qu'on a

$$
\left.\frac{\partial \rho_{0}(x, \eta)}{\partial n}\right|_{x=0}=\left(2 \sum_{j=1}^{2} \frac{\eta_{j}^{2}}{R_{j}}\right)^{1 / 3}
$$

d'après la méthode de construction de $\theta_{0}$ et de $\rho_{0}$, où $R_{1}$ et $R_{2}$ sont les courbures principales de $\Gamma$ en $s=0$.

Désignons par $L^{+}(s, \eta)\left(L^{-}(s, \eta)\right)$ pour $s \in \Gamma_{0}$ et $\eta \in \Sigma$ une demi-droite s'etendant de $s$ à la direction $\nabla \theta_{0}(s, \eta)\left(-\nabla \theta_{0}(s, \eta)\right)$ et par $L(s, \eta)$ une droite $L^{+}(s, \eta) \cup L^{-}(s, \eta)$. Si l'on corresponde pour $x \in \bar{\Omega}$ un pair $\{s, r\} \in \Gamma \times \overline{\mathbb{R}_{+}^{1}}$ de façon que $x$ soit sur la normale de $\Gamma$ passant $s$ et que la distance entre $x$ et $s$ soit $r$, on a une application bijective de $\bar{\Omega}$ dans $\Gamma \times \overline{\boldsymbol{R}_{+}^{1}}$ d'après la convexité stricte de $\mathcal{O}$. Nous désignons par $\{s, r\}$ un point $x \in \bar{\Omega}$ selon la nécessité. Et nous posons

$$
\Gamma_{j} \times\left[r_{1}, r_{0}\right]=\left\{x ; x=\{s, r\}, s \in \Gamma_{j}, r_{1} \leq r \leq r_{0}\right\}, j=0,1 .
$$

En posant

$$
\psi_{0}^{ \pm}(x, \eta)=\theta_{0}(x, \eta) \pm \frac{2}{3} \rho_{0}(x, \eta)^{3 / 2}
$$

on a $\psi_{0}^{ \pm}(x, \eta) \in C^{\infty}(\Omega \cap q i)$ et

$$
\left|\nabla \psi_{0}^{ \pm}\right|^{2}=1 \quad \text { dans } \bar{\Omega} \cap \mathcal{U} .
$$

Notons que $\psi_{0}^{ \pm}(x, \eta)$ peut être prolongée à une fonction définie dans $\bigcup_{s \in \Gamma_{0}} L^{+}(s, \eta)$ et vérifiant (3.5). A propos de ces $\theta_{0}, \rho_{0}$ on a le

Lemme 3. 1. Soient $\gamma(x), \delta(x) \in \mathscr{B}\left(\mathbb{R}^{3}\right)$. Le système des équations

$$
\begin{array}{r}
2 \nabla \theta_{0} \cdot \nabla f_{0}+2 \rho_{0} \nabla \rho_{0} \cdot \nabla f_{1}+\left(\nabla \rho_{0}\right)^{2} f_{1}+\rho_{0} \delta(x) f_{1}+\gamma(x) f_{0}=h_{0} \\
\text { dans } \bar{\Omega} \cap U
\end{array}
$$




$$
\begin{gathered}
2 \nabla \rho_{0} \cdot \nabla f_{0}+2 \nabla \theta_{0} \cdot \nabla f_{1}+\delta(x) f_{0}+\gamma(x) f_{1}=h_{1} \quad \text { dans } \bar{\Omega} \cap \mathcal{U} \\
f_{0}(s)=m(s) \quad \text { sur } \Gamma_{0}
\end{gathered}
$$

ademt une solution unique $\left\{f_{0}, f_{1}\right\} \in C^{\infty}(\bar{\Omega} \cap \mathcal{U})$ pour les données $h_{0}, h_{1} \in C^{\infty}(\bar{\Omega} \cap \mathcal{U}), m(s) \in C^{\infty}\left(\Gamma_{0}\right)$, et l'application $\left\{h_{0}, h_{1}, m\right\} \rightarrow\left\{f_{0}, f_{1}\right\}$ est continue de $C^{\infty}(\bar{\Omega} \cap \mathcal{U}) \times C^{\infty}(\bar{\Omega} \cap \mathcal{U}) \times C^{\infty}\left(\Gamma_{0}\right)$ dans $C^{\infty}(\bar{\Omega} \cap \mathcal{U})$ $\times C^{\infty}(\bar{\Omega} \cap \mathcal{U}) . \quad$ Et plus

$$
\bigcup_{j=0}^{1} \operatorname{supp} h_{j} \subset \bigcup_{\substack{s \in \text { supp } m \\ \eta \in \Sigma}} L(s, \eta)
$$

entaîne

$$
\bigcup_{j=0}^{1} \operatorname{supp} f_{j} \subset \underset{\substack{s \in \operatorname{supp}_{\eta \in \Sigma} m \\ \eta \in \Sigma}}{ } L(s, \eta)
$$

Donc selon le raisonnement de $\S 5$ de [4] nous pouvons construire des fonctions $\theta(x, \eta, \beta), \rho(x, \eta, \beta)$ définies pour $x \in \mathcal{U}, \eta \in \Sigma, \beta \in I_{\beta}$ $=\left[-\beta_{0}, \beta_{0}\right]$ et vérifiant

$$
\left\{\begin{array}{l}
(\nabla \theta)^{2}+\rho(\nabla \rho)^{2} \equiv 1 \quad\left(\bmod \beta^{\infty}\right) \quad \text { dans } U \cap \bar{\Omega} \\
\nabla \theta \cdot \nabla \rho \equiv 0 \quad\left(\bmod \beta^{\infty}\right) \quad \text { dans } \bar{\Omega} \cap \mathcal{U} \\
\rho=-\beta \quad \text { sur } \Gamma \cap \mathcal{U} \\
\theta(s, \eta, 0)=\theta_{0}(s, \eta) \quad s \in \Gamma \cap U, \eta \in \Sigma .
\end{array}\right.
$$

Alors par le processus de la construction on voit que

$$
\begin{aligned}
& (\nabla \theta)(0, \eta, 0)=\eta_{1} \frac{\partial}{\partial \sigma_{1}}+\eta_{2} \frac{\partial}{\partial \sigma_{2}} \\
& \frac{\partial^{2} \theta}{\partial \beta \partial \sigma_{j}}(0, \eta, 0)=\eta_{j}\left(2 \sum_{j=1}^{2} \frac{\eta_{j}^{2}}{R_{j}}\right)^{1 / 3} .
\end{aligned}
$$

Posons

$$
\begin{aligned}
& F\left(\sigma, \sigma^{\prime}, \eta, \beta, \xi^{\prime}, \alpha\right)=\theta(s(\sigma), \eta, \beta)-\theta\left(s\left(\sigma^{\prime}\right), \eta, \beta\right) \\
& -\varphi(s(\sigma))+\varphi\left(s\left(\sigma^{\prime}\right)\right)+\left\langle\sigma-\sigma^{\prime}, \varphi^{0}\right\rangle-(1+\alpha)\left\langle\sigma-\sigma^{\prime}, \xi^{\prime}\right\rangle
\end{aligned}
$$

et

$$
F\left(\sigma, \sigma^{\prime}, \eta, \beta, \xi^{\prime}, \alpha\right)=\sum_{j=1}^{2}\left(\sigma_{j}-\sigma_{j}^{\prime}\right) F_{j}\left(\sigma, \sigma^{\prime}, \eta, \beta, \xi^{\prime}, \alpha\right)
$$




$$
F_{3}\left(\sigma, \sigma^{\prime}, \eta, \beta, \xi^{\prime}, \alpha\right)=\eta_{1}^{2}+\eta_{2}^{2}-1
$$

Alors on a d'après (3.8)

$$
\begin{aligned}
\left.\frac{D\left(F_{1}, F_{2}, F_{3}\right)}{D\left(\eta_{1}, \eta_{2}, \beta\right)}\right|_{\substack{\beta=\alpha=0 \\
\sigma=\sigma^{\prime}=0 \\
\eta=\xi^{\prime}}} & =\operatorname{dét}\left[\begin{array}{ccc}
\frac{\partial^{2} \theta}{\partial \eta_{1} \partial \sigma_{1}} & \frac{\partial^{2} \theta}{\partial \eta_{2} \partial \sigma_{1}} \frac{\partial^{2} \theta}{\partial \beta \partial \sigma_{1}} \\
\frac{\partial^{2} \theta}{\partial \eta_{1} \partial \sigma_{2}} & \frac{\partial^{2} \theta}{\partial \eta_{2} \partial \sigma_{2}} \frac{\partial^{2} \theta}{\partial \beta \partial \sigma_{2}} \\
\eta_{1} & \eta_{2} & 0
\end{array}\right]_{\substack{\sigma^{\prime}=0 \\
\beta=0}} \\
& =-\left(\nabla \rho_{0}(0, \eta, 0)\right)^{2} \neq 0 .
\end{aligned}
$$

Il est évident qu'il a lieu

$$
F_{j}(0,0, \eta, 0, \eta, 0)=0, \quad \forall \eta \in \Sigma, \quad j=1,2,3 .
$$

Donc on trouve des fonctions $\eta_{j}\left(\sigma, \sigma^{\prime}, \xi^{\prime}, \alpha\right), j=1,2$ et $\beta\left(\sigma, \sigma^{\prime}, \xi^{\prime}, \alpha\right)$ définies sur $I_{\sigma} \times I_{\sigma} \times \Sigma \times I_{\alpha}$ telles que

$$
F_{j}\left(\sigma, \sigma^{\prime}, \eta, \beta, \xi^{\prime}, \alpha\right)=0, \quad j=1,2,3, \quad \forall\left(\sigma, \sigma^{\prime}, \xi^{\prime}, \alpha\right) \in I_{\sigma} \times I_{\sigma} \times \Sigma \times I_{\alpha}
$$

en choisissant $I_{\sigma}, I_{\alpha}$ petits s'il est nécessaire. Cela signifie

$$
\begin{gathered}
\theta(s(\sigma), \eta, \beta)-\theta\left(s\left(\sigma^{\prime}\right), \eta, \beta\right)-\varphi(s(\sigma))+\varphi\left(s\left(\sigma^{\prime}\right)\right) \\
=-\left\langle\sigma-\sigma^{\prime}, \varphi^{0}\right\rangle+(1+\alpha)\left\langle\sigma-\sigma^{\prime}, \xi^{\prime}\right\rangle, \\
\eta_{1}^{2}+\eta_{2}^{2}=1 .
\end{gathered}
$$

Puisque $\eta\left(0,0, \xi^{\prime}, 0\right)=\xi^{\prime}, \quad \beta\left(0,0, \xi^{\prime}, 0\right)=0, \quad \frac{\partial \beta}{\partial \alpha}\left(0,0, \xi^{\prime}, 0\right)$ $=(\nabla \rho(0, \eta, 0))^{-2}$ il existe la fonction inverse de

$$
\begin{aligned}
& \eta\left(\sigma, \sigma^{\prime}, \xi^{\prime}, \alpha\right)=\eta \\
& \beta\left(\sigma, \sigma^{\prime}, \xi^{\prime}, \alpha\right)=\beta
\end{aligned}
$$

pour $\eta \in \Sigma, \beta \in I_{\beta}$. Prenons $\alpha_{0}>0, \beta_{0}>0$ de telle façon qu'il ait lieu

$$
I_{\beta} \subset\left\{\beta\left(\sigma, \sigma^{\prime}, \xi^{\prime}, \alpha\right) ; \alpha \in I_{\alpha}\right\}
$$

pour tout $\left(\sigma, \sigma^{\prime}, \xi\right) \in I_{\sigma} \times I_{\sigma} \times \Sigma$. Désignons cette fonction inverse par

$$
\alpha=\alpha\left(\sigma, \sigma^{\prime}, \eta, \beta\right), \quad \xi^{\prime}=\xi^{\prime}\left(\sigma, \sigma^{\prime}, \eta, \beta\right)
$$

Alors il a lieu

$$
\left.\frac{D\left(\xi^{\prime}, \alpha\right)}{D(\eta, \beta)}\right|_{\substack{\sigma=\sigma^{\prime}=0 \\ \eta=\xi^{\prime} \\ \alpha=\beta=0}}=(\nabla \rho(0, \eta, 0))^{2} .
$$


Donc on a

$$
\begin{aligned}
& V_{2} g(s(\sigma))= \lambda(\sigma) \int_{I_{\alpha}} d \alpha \int_{\Sigma} d \xi^{\prime} \int_{I_{\sigma}} d \sigma^{\prime} \exp \left[i k \left\{-\left\langle\sigma-\sigma^{\prime}, \varphi^{0}\right\rangle\right.\right. \\
&\left.\left.+(1+\alpha)\left\langle\sigma-\sigma^{\prime}, \xi^{\prime}\right\rangle\right\}\right] \chi_{2}(1+\alpha)^{2} k^{2}(1+\alpha) g\left(s\left(\sigma^{\prime}\right)\right) \\
&=\lambda(\sigma) \int_{I_{\beta}} d \beta \int_{\Sigma} d \eta \int_{I_{\sigma}} d \sigma^{\prime} \exp \left[i k \left\{\theta(s(\sigma), \eta, \beta)-\theta\left(s\left(\sigma^{\prime}\right), \eta, \beta\right)\right.\right. \\
&\left.\left.-\varphi(s(\sigma))+\varphi\left(s\left(\sigma^{\prime}\right)\right)\right\}\right] \chi_{2}\left(1+\alpha\left(\sigma, \sigma^{\prime}, \eta, \beta\right)\right)^{2} \\
& \times k^{2}\left(1+\alpha\left(\sigma, \sigma^{\prime}, \eta, \beta\right)\right) \frac{D\left(\xi^{\prime}, \alpha\right)}{D(\eta, \beta)} g\left(s\left(\sigma^{\prime}\right)\right) .
\end{aligned}
$$

Prenons des fonctions à valeurs réelles $v_{j}(l) \in C^{\infty}\left(\boldsymbol{R}^{1}\right), j=1,2,3$ de telle façon que

$$
v_{1}(l)=\left\{\begin{array}{ll}
1 & l<-2 \\
0 & l>-1
\end{array}, \quad v_{2}(l)=\left\{\begin{array}{ll}
1 & |l| \leq 1 \\
0 & |l| \geq 2
\end{array}, \quad v_{3}(l)= \begin{cases}1 & l>2 \\
0 & l<1\end{cases}\right.\right.
$$

et que $\sum_{j=1}^{3} v_{j}(l)^{2}=1$.

Définissons $V_{2 j}, j=1,2,3$ par

$$
\begin{aligned}
V_{2 j} g(s(\sigma)) & =\lambda(\sigma) \int_{I_{\beta}} d \beta \int_{\Sigma} d \eta \int_{I_{\sigma}} d \sigma^{\prime} \exp [i k\{\theta(s(\sigma), \eta, \beta) \\
& \left.\left.-\theta\left(s\left(\sigma^{\prime}\right), \eta, \beta\right)-\varphi(s(\sigma))+\varphi\left(s\left(\sigma^{\prime}\right)\right)\right\}\right] \chi_{2}(1+\alpha)^{2}(1+\alpha) \\
& \times k^{2} v_{j}\left(k^{\varepsilon} \beta\right)^{2} \frac{D\left(\xi^{\prime}, \alpha\right)}{D(\eta, \beta)} g\left(s\left(\sigma^{\prime}\right)\right),
\end{aligned}
$$

où $\varepsilon$ est une constante positive, qui sera déterminée plus tard.

Il est évident que

$$
V_{2} g=\sum_{j=1}^{3} V_{2 j} g .
$$

D'après la définitions de $\chi_{2}$ et de $v_{2}$ il a lieu

$$
\chi_{2}\left(1+\alpha\left(\sigma, \sigma^{\prime}, \eta, \beta\right)\right)^{2} v_{2}\left(k^{\varepsilon} \beta\right)^{2}=v_{2}\left(k^{\varepsilon} \beta\right)^{2}, \quad \forall \beta \in I_{3}
$$

pour $k$ suffisamment grand.

Nous allons construire $U_{2 j}(p, g: x), j=1,2,3$ vérifiant asymptotiquement par rapport à $|p|$

$$
\left\{\begin{array}{l}
A_{\varphi}(p, \partial / \partial x) U_{2 j}(p, g ; x)=0 \quad \text { dans } \quad \Omega \\
\left.U_{2 j}(p, g: x)\right|_{\Gamma}=V_{2 j} g(x) \quad \text { sur } \quad \Gamma .
\end{array}\right.
$$




\section{$\S 4$. Construction de $\boldsymbol{U}_{22}$}

D'abord considérons un problème aux limites avec une donnée au bord oscillatoire

$$
\left\{\begin{array}{l}
A_{\varphi}(p, \partial / \partial x) u(x)=0 \quad \text { dans } \Omega \\
u(x)=\exp \{i k(\theta(s, \eta, \beta)-\varphi(s))\} v(s) \quad \text { sur } \Gamma,
\end{array}\right.
$$

où $v(s) \in \mathscr{D}\left(\Gamma_{1}\right)$. Nous cherchons une solution asymptotique de (4.1) sous la forme

$$
\begin{aligned}
u(x: & : p, \eta, \beta)=\exp \{i k(\nu(x, \eta, \beta, p)-\varphi(x))\} \\
& \times \frac{1}{H\left(k^{2 / 3} \hat{\zeta}(x, \eta, \beta, p)\right)}\left\{H \left(k^{2 / 3} \zeta(x, \eta, \beta, p) g_{0}\right.\right. \\
& \left.+\frac{1}{i k^{1 / 3}} H^{\prime}\left(k^{2 / 3} \zeta(x, \eta, \beta, p)\right) g_{1}\right\}
\end{aligned}
$$

où

$$
H(z)=A i(-z)+i B i(-z)
$$

et $\grave{\zeta}(x, \eta, \beta, p)$ est définie $\operatorname{par} \zeta(s, \eta, \beta, p)$ en désignant $x=\{s, r\}$. Appliquer $A_{\varphi}$ à $u$ de (4.2).

$$
\begin{aligned}
\exp \{- & i k(\nu-\varphi)\} \cdot H\left(k^{2 / 3} \widehat{\zeta}\right)^{-1} A_{\varphi}(p, \partial / \partial x) u \\
= & H\left(k^{2 / 3} \zeta\right)\left[\left\{p^{2}-(i k)^{2}\left\{\left(\nabla \nu+\frac{\mu}{i k} \nabla \varphi-\frac{1}{i k^{1 / 3}} R \nabla \hat{\zeta}\right)^{2}+\zeta(\nabla \zeta)^{2}\right\}\right\} g_{0}\right. \\
& -(i k)^{2}\left(\nabla \nu+\frac{\mu}{i k} \nabla \varphi-\frac{1}{i k^{1 / 3}} R \nabla \widehat{\zeta}\right) \zeta \nabla \zeta \cdot g_{1} \\
& -i k\left\{2\left(\nabla \nu+\frac{\mu}{i k} \nabla \varphi-\frac{1}{i k^{1 / 3}} R \nabla \hat{\zeta}\right) \nabla g_{0}+\nabla\left(\nabla \nu+\frac{\mu}{i k} \nabla \varphi\right.\right. \\
& \left.\left.\left.-\frac{1}{i k^{1 / 3}} R \nabla \hat{\zeta}\right) g_{0}+2 \zeta \nabla \zeta \cdot \nabla g_{1}+\nabla(\zeta \nabla \zeta) g_{1}\right\}-\Delta g_{0}\right] \\
& +\frac{1}{i k^{1 / 3}} H^{\prime}\left(k^{2 / 3} \zeta\right)\left[\left\{p^{2}-(i k)^{2}\left\{\left(\nabla \nu+\frac{\mu}{i k} \nabla \varphi-\frac{1}{i k^{1 / 3}} R \nabla \hat{\zeta}\right)^{2}\right.\right.\right. \\
& \left.\left.+\zeta(\nabla \zeta)^{2}\right\}\right\} g_{1}-(i k)^{2}\left(\nabla \nu+\frac{\mu}{i k} \nabla \varphi-\frac{1}{i k^{1 / 3}} R \nabla \hat{\zeta}\right) \nabla \zeta \cdot g_{0}
\end{aligned}
$$




$$
\begin{aligned}
& -i k\left\{2\left(\nabla \nu+\frac{\mu}{i k} \nabla \varphi-\frac{1}{i k^{1 / 3}} R \nabla \hat{\zeta}\right) \nabla g_{1}+\nabla\left(\nabla \nu+\frac{\mu}{i k} \nabla \varphi\right.\right. \\
& \left.\left.\left.-\frac{1}{i k^{1 / 3}} R \nabla \hat{\zeta}\right) g_{1}+2 \nabla \zeta \cdot \nabla g_{0}+\Delta \zeta \cdot g_{0}\right\}-\Delta g_{1}\right] .
\end{aligned}
$$

Donc nous cherchons $\nu$ et $\zeta$ de façon qu'il ait lieu

$$
\begin{array}{ll} 
& \left(\bmod \left(|p|^{-1}+|\beta|\right)^{\infty}\right) \quad \text { dans } \bar{\Omega} \cap \mathcal{U} \\
\nu(s, \eta, \beta, p)=\theta(s, \eta, \beta) & \text { sur } \Gamma \cap \mathcal{}
\end{array}
$$$$
\left(\bmod |p|^{-\infty} \beta^{\infty}\right) \quad \text { dans } \quad \bar{\Omega} \cap \mathcal{U}
$$

et puis $g_{0}$ et $g_{1}$ de façon que

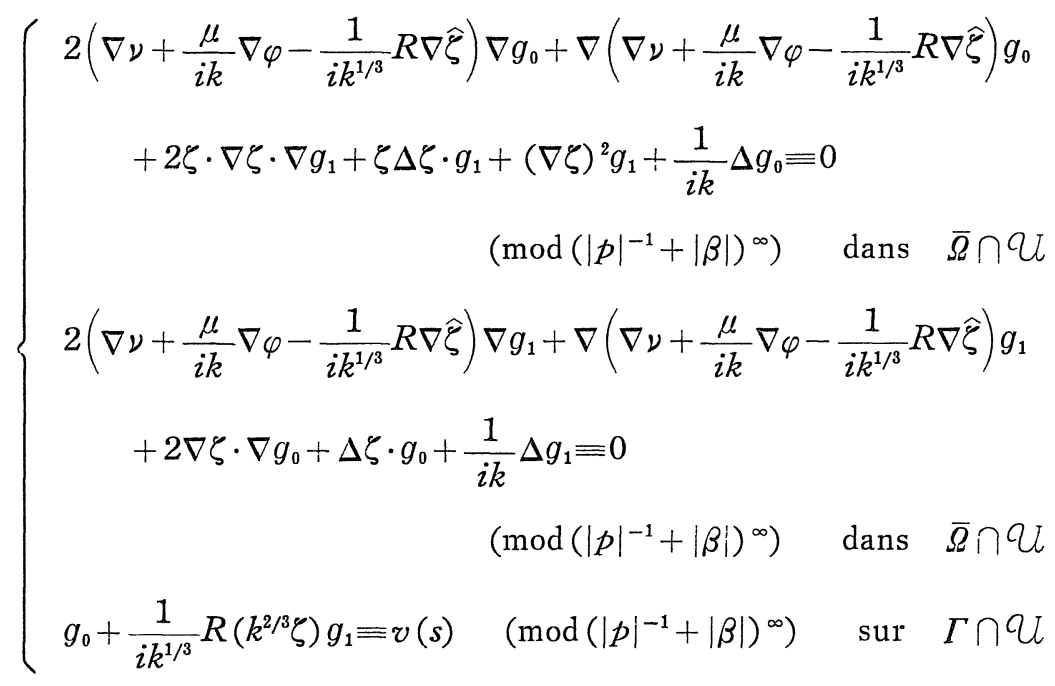

où $R(z)=\frac{H^{\prime}(z)}{H(z)}$.

Les constructions de $\nu$, de $\zeta$, de $g_{0}$ et de $g_{1}$ peuvent être faites selon le raisonnement de $\S 5$ de [4] sous la restriction

$$
0<\mu \leq a_{0} k^{1 / 3}
$$

où $a_{0}$ est une constante assez petite. Notons qu'il a lieu

$$
g_{j}(x, \eta, \beta, p) \in S_{1 / 3,0}^{0}(\bar{\Omega} \cap \mathcal{U}), \quad j=0,1
$$


et que $\zeta$ est développée sur $\Gamma_{0}$ comme suit:

(4. 6) $\quad \zeta(s, \eta, \beta, p)$

$$
=-\beta+\frac{\mu}{i k}\left\{\frac{2(1-\nabla \varphi(s) \cdot \nabla \theta(s, \eta, 0))}{(\nabla \rho(s, \eta, 0))^{2}}+0\left(|\beta|+\frac{\mu}{k}\right)\right\} .
$$

Alors la fonction $u(x, \eta, \beta, p)$ définie par (4.2) avec ces fonctions satisfait à

$$
\left\{\begin{array}{rr}
A_{\varphi}(p, \partial / \partial x) u \equiv 0 \quad\left(\bmod \left(|p|^{-1}+|\beta|\right)^{\infty}\right) & \text { dans } \quad \bar{\Omega} \cap \mathcal{L}_{l} \\
u(s, \eta, \beta, p) \equiv \exp \{i k(\theta(s, \eta, \beta)-\varphi(s))\} \cdot v(s) & \\
\left(\bmod \left(|p|^{-1}+|\beta|\right)^{\infty}\right) & \text { sur } \quad \Gamma \cap \mathcal{U}_{l}
\end{array}\right.
$$

Supposons que pour $0<r_{0}<r_{1}$

$$
\Gamma_{0} \times\left[0, r_{1}\right] \subset \mathcal{U}
$$

et que

$$
\bigcup_{\substack{s \in \Gamma_{1} \\ \eta \in \Sigma}} L(s, \eta) \cap \Gamma \times\left[0, r_{1}\right] \subset \Gamma_{0} \times\left[0, r_{1}\right]
$$

Alors puisqu'il a lieu pour $x \in \Gamma_{0} \times\left[r_{0}, r_{1}\right]$

$$
\begin{array}{lc}
\operatorname{Re} k^{2 / 3} \zeta(x, \eta, \beta, p) \rightarrow \infty & \text { lorsque } \quad k \rightarrow \infty \\
\left|\operatorname{Im} k^{2 / 3} \zeta(x, \eta, \beta, p)\right| \leq C a_{0} \quad \forall k>0,
\end{array}
$$

$u(x, \eta, \beta, p)$ s'exprime, en utilisant le comportement asymptotique de $H(z)$ pour $\operatorname{Re} z \rightarrow \infty$, comme

$$
u(x, \eta, \beta, p)=\exp \left\{i k\left(\nu-\varphi+\frac{2}{3} \zeta^{3 / 2}\right)\right\} \cdot H\left(k^{2 / 3} \widehat{\xi}\right)^{-1} \cdot G^{+}(x, \eta, \beta, p)
$$

où on a $G^{+} \in S_{1 / 3,0}^{0}(\bar{\Omega} \cap \mathcal{Q})$. Et en posant

$$
\psi^{+}(x, \eta, \beta, p)=\nu+\frac{2}{3} \zeta^{3 / 2}-\frac{1}{k} \log H\left(k^{2 / 3} \widehat{\zeta}\right)
$$

il a lieu

$$
\psi^{+} \in S_{1 / s, 0}^{0}\left(\Gamma_{0} \times\left[r_{0}, r_{1}\right]\right) .
$$

Grâce à la méthode de construction de $g_{0}$ et de $g_{1}$ pour tout $\tilde{I}_{1}$ voisinage du support de $v$ il existe $r_{0}>0$ tel que $g_{0}$ et $g_{1}$ s'annulent dans $\left(\Gamma-\widetilde{\Gamma}_{1}\right) \times\left[0, r_{0}\right]$. Donc 


$$
G^{+}=0 \quad \text { dans } \quad\left(\underset{\substack{s \in \Gamma-\widetilde{T}_{1} \\ \eta \in \Sigma}}{\bigcup} L(s, \eta)\right) \cap \Gamma \times\left[0, r_{0}\right] .
$$

Notons que $G^{+}$est indéfiniment différentiable par rapport à $k^{-1}$, à $\left(-\frac{\mu}{i k}\right)$ et à $\beta$. Supposons que $G^{+}$se développe dans $\Gamma_{0} \times\left[r_{0}, r_{1}\right]$ comme

$$
G^{+} \sim \sum G_{j l m}^{\vdash}(x, \eta) k^{-j}\left(\frac{\mu}{i k}\right)^{l} \beta^{m},
$$

et cherchons les relations que les $G_{j l m}^{+}(x, \eta)$ doivent satisfaire. D'après (4.3) et la définition de $\psi^{+}$, nous avons

$$
\begin{aligned}
\left(\nabla \psi^{+}+\right. & \left.\frac{\mu}{i k} \nabla \varphi\right)^{2} \equiv\left(1+\frac{\mu}{i k}\right)^{2} \\
& \left(\bmod \left(\left|p^{\prime-1}+\right| \beta \mid\right)^{\infty}\right) \text { dans } \Gamma_{0} \times\left[r_{0}, r_{1}\right] .
\end{aligned}
$$

Puisque $\psi^{+}$est indéfiniment différentiable par rapport à $\left(\frac{\mu}{i k}\right)$ et à $\beta$, nous avons

$$
\psi^{+} \sim \sum_{j, l} \psi_{j l}^{+}(x, \eta) \beta^{j}\left(\frac{\mu}{i k}\right)^{l}
$$

La substitution du développement à (4.10) nous donne

$$
\left\{\begin{array}{l}
\left(\nabla \psi_{00}^{+}\right)^{2}=1 \\
2 \nabla \psi_{00}^{+} \nabla \psi_{j l}^{+}+\sum_{(\{p, q\},\{r, s\}) \in \mathscr{T}\{, l} \nabla \psi_{p q}^{+} \cdot \nabla \psi_{r s}^{+}=N_{l} \quad j+l \geq 1
\end{array}\right.
$$

où $N_{1}=2, N_{2}=1$ et $N_{r}=0$ pour $r \geq 3$, et $\mathcal{H}_{j, l}=\{(\{p, q\},\{r, s\}) ; p+r=l$, $\left.q+s=j, \quad\{p, q\},\{r, s\} \in \mathcal{I}_{j+l+1}\right\}, \mathcal{G}_{k}=\{\{p, q\} ; p+q \leq k\}$.

Notons que $\psi_{00}^{+}(x, \eta)=\psi^{+}(x, \eta, 0, \mu+i \infty)=\theta_{0}(x, \eta)+\frac{2}{3} \rho_{0}(x, \eta)^{3 / 2}$ est définie sur $\bigcup_{s \in \Gamma_{0}} L^{+}(s, \eta)$ et qu'une équation

$$
\left\{\begin{array}{l}
\nabla \psi_{00}^{+} \cdot \nabla f=h(x) \quad \text { dans } \bigcup_{s \in \Gamma_{0}} L^{+}(s, \eta) \cap \Gamma \times\left[r_{0}, \infty\right) \\
\left.f\right|_{\Gamma_{0} \times 0}=m(x)
\end{array}\right.
$$

admet une solution unique dans $C^{\infty}\left(\cup L^{+}(s, \eta) \cap \Gamma \times\left[r_{0}, \infty\right)\right)$ pour les données $h, m \in C^{\infty}$. Donc les fonctions $\psi_{j l}(x, \eta), j+l \geq 1$, qui sont définies sur $\Gamma_{0} \times\left[r_{0}, r_{1}\right]$, peuvent être prolongées en $\bigcup_{s \in \Gamma_{0}} L^{+}(s, \eta) \cap \Gamma \times\left[r_{0}, \infty\right)$ par les relation (4.11). Ainsi nous pouvons supposer que la fonction $\psi^{+}$ est définie sur $\bigcup_{s \in \Gamma_{0}} L^{+}(s, \eta) \cap \Gamma \times\left[r_{0}, \infty\right)$ et vérifies

$$
\left(\nabla \psi^{+}+\frac{\mu}{i k} \nabla \varphi\right)^{2}=\left(1+\frac{\mu}{i k}\right)^{2}\left(\bmod \left(|p|^{-1}+|\beta|\right)^{\infty}\right)
$$




$$
\text { dans } \bigcup_{s \in \Gamma_{0}} L^{+}(s, \eta) \cap \Gamma \times\left[r_{0}, \infty\right)
$$

D'après $A_{\varphi}\left(\exp \left\{i k \psi^{+}\right\} \cdot G^{+}\right) \equiv 0$ dans $\Gamma \times\left[r_{0}, r_{1}\right]$ nous avons

$$
2 \nabla \psi^{+} \cdot \nabla G^{+}+\Delta \psi^{+} \cdot G^{+}+\frac{1}{i k} \Delta G^{+} \equiv 0 .
$$

Par la substitution de (4.9) en relation au-dessus nous donne des équations que $G_{j l m}^{+}$doivent vérifier et $G_{j l m}^{+}$sont prolongées dans $\bigcup_{s \in \Gamma_{0}} L^{+}(s, \eta)$ $\cap \Gamma \times\left[r_{0}, \infty\right)$ en satisfaisant aux équations. Donc $G^{+}$peut être prolongée de façon que

$$
\begin{aligned}
& A_{\varphi}\left(\exp \left\{i k \psi^{+}\right\} \cdot G^{+}\right) \equiv 0 \quad \text { dans } \quad \Gamma \times\left[r_{0}, \infty\right) . \\
& \frac{\partial}{\partial r}\left(\exp \{i k(\nu-\varphi)\} \cdot H\left(k^{2 / 3} \zeta\right) \cdot H\left(k^{2 / 3} \xi\right)^{-1}\right) \\
& =i k\left\{\frac{\partial \nu}{\partial r}-\frac{\partial \varphi}{\partial r}+\frac{1}{i k^{1 / 3}}\left(R\left(k^{2 / 3} \zeta\right) \frac{\partial \zeta}{\partial r}-R\left(k^{2 / 3} \widehat{\zeta}\right) \frac{\partial \widehat{\zeta}}{\partial r}\right)\right\} \\
& \times \exp \{i k(\nu-\varphi)\} \cdot H\left(k^{2 / 3} \zeta\right) \cdot H\left(k^{2 / 3} \widehat{\zeta}\right)^{-1},
\end{aligned}
$$

et

$$
-\operatorname{Re} i k\left\{\frac{\partial \nu}{\partial r}-\frac{\partial \varphi}{\partial r}+\frac{1}{i k^{1 / 3}}\left(R\left(k^{2 / 3} \zeta\right) \frac{\partial \zeta}{\partial r}-R\left(k^{2 / 3} \widehat{\zeta}\right) \frac{\partial \widehat{\zeta}}{\partial r}\right)\right\} \geq c_{0} \mu
$$

donc nous avons

$$
\begin{gathered}
\left|\exp \{i k(\nu-\varphi)\} H\left(k^{2 / 3} \zeta\right) \cdot H\left(k^{2 / 3} \widehat{\zeta}\right)^{-1}\right| \leq \exp \left(-c_{0} \mu r\right) \\
\text { pour } 0 \leq r \leq r_{0}
\end{gathered}
$$

et d'après (4.11)

$$
\left|\exp \left(i k \psi^{+}\right)\right| \leq \exp \left\{-c_{0}^{\prime} \mu\left(r-r_{0}\right)\right\} \exp \left(-c_{0} \mu r_{0}\right) .
$$

D'autre part, les équations qui déterminent $g_{0}, g_{1}$ et $G^{+}$montrent qu'il existe une constante $c^{\prime}>0$ telle que

$$
\begin{array}{lll}
\left|g_{j}\right| \leq C \cdot \exp \left(c^{\prime} r\right) \quad \text { pour } & 0 \leq r \leq r_{0} \\
\left|G^{+}\right| \leq C \exp \left\{c^{\prime}\left(r-r_{0}\right)\right\} & \text { pour } \quad r \geq r_{0} .
\end{array}
$$

De cette façon nous avons

$$
\left|A_{\varphi} u\right| \leq C_{N} \exp (-c \mu r) \cdot\left(|\beta|+\left|\frac{\mu}{k}\right|\right)^{N} \quad \forall x \in \bar{\Omega} .
$$


Définissons $U_{22}(p, g: x)$. Pour une fonction

$$
v(s)=\lambda(s)\left(1+\alpha\left(s, s^{\prime}, \eta, \beta\right)\right) \frac{D\left(\xi^{\prime}, \alpha\right)}{D(\eta, \beta)}\left(s, s^{\prime}, \eta, \beta\right)
$$

avec les paramètres $s^{\prime}, \eta, \beta$, construisons les fonctions $g_{j}\left(x, s^{\prime}, \eta, \beta, p\right)$, $j=0,1$ et $G^{+}\left(x, s^{\prime}, \eta, \beta, p\right)$ selon la méthode jusqu'à maintenant. Remarquons que les supports de $g_{j}$ et de $G^{+}$sont contenus dans $\underset{s \in \Gamma_{0}}{\cup} L(s, \eta)$. En posant $g_{j}\left(x, s^{\prime}, \eta, \beta, p\right)=0, G^{+}=0$ dans $\bar{\Omega}-\bigcup_{s \in \Gamma_{0}} L^{+}(s, \eta)$, on a $g_{j}$ et $G^{+} \in C^{\infty}(\bar{\Omega})$. Définissons

$$
\begin{aligned}
& U_{22}(p, g: x)=\int_{I_{\beta}} d \beta \int_{\Sigma} d \eta \int_{I_{\sigma}} d \sigma^{\prime} \cdot \exp \left\{i k\left(\theta\left(s\left(\sigma^{\prime}\right), \eta, \beta\right)-\varphi\left(s\left(\sigma^{\prime}\right)\right)\right)\right\} \\
& \quad \times\left[\operatorname { e x p } \{ i k ( \nu ( x , \eta , \beta , p ) - ( \varphi x ) ) \} \cdot H ( k ^ { 2 / 3 } \widehat { \zeta } ) ^ { - 1 } \left\{H\left(k^{2 / 3} \zeta(x, \eta, \beta, p)\right)\right.\right. \\
& \quad \times g_{0}\left(x, s\left(\sigma^{\prime}\right), \eta, \beta, p\right)+\frac{1}{i k^{1 / 3}} H^{\prime}\left(k^{2 / 3} \zeta(x, \eta, \beta, p)\right) \\
& \left.\quad \times g_{1}\left(x, s\left(\sigma^{\prime}\right), \eta, \beta, p\right)\right\} u_{2}\left(\frac{r}{r_{0}}\right)^{2}+\exp \left\{i k\left(\psi^{+}(x, \eta, \beta, p)-\varphi(x)\right)\right\} \\
& \left.\quad \times G^{+}\left(x, s\left(\sigma^{\prime}\right), \eta, \beta, p\right) \cdot v_{3}\left(\frac{r}{r_{0}}\right)^{2}\right] u_{2}\left(k^{\varepsilon} \beta\right)^{2} k^{2} g\left(s\left(\sigma^{\prime}\right)\right) .
\end{aligned}
$$

Alors $U_{22}(p, g: x) \in C^{\infty}(\bar{\Omega})$ et $\operatorname{supp} U_{22} \subset \underset{\substack{s \in \Gamma^{0} \\ \eta \in \Sigma^{0}}}{ } L^{+}(s, \eta)$.

En tenant compte de $|\beta| \leq k^{-\varepsilon}$ on déduit de (4.12)

$$
\begin{aligned}
& \left\|A_{\varphi} U_{22}(p, g: x)\right\|_{m} \leq C_{m, m^{\prime}, N}|p|^{-N}\|g\|_{m^{\prime}} \\
& \left\|\left.U_{22}(p, g: x)\right|_{\Gamma}-V_{22} g\right\|_{m} \leq C_{m, m^{\prime}, N}|p|^{-N}\|g\|_{m^{\prime}}
\end{aligned}
$$

pour tous $m, m^{\prime}, N$ les constantes non-négatives.

Donc nous avons la

Proposition 4.1. Pour $p=i k+\mu$ tel que $\mu \geq \mu_{\varphi}, \mu \leq a_{0} k^{1 / 3} \quad$ 'application $U_{22}$ de $\mathscr{D}\left(\Gamma_{1}\right)$ dans $C^{\infty}(\bar{\Omega})$ est continue et satisfait à (4.14) et $\grave{a}$ (4.15).

$\S$ 5. $\operatorname{Sur} \frac{\partial U_{22}}{\partial n}$

Par le définition de $U_{22}$ nous avons 
(5. 1) $\left.\frac{\partial U_{22}}{\partial n}\right|_{\Gamma}=\int_{I_{\beta}} d \beta \int_{\Sigma} d \eta \int_{I_{\sigma}} d \sigma^{\prime} \exp \left[i k\left\{\theta(s(\sigma), \eta, \beta)-\theta\left(s\left(\sigma^{\prime}\right), \eta, \beta\right)\right.\right.$

$$
\begin{aligned}
& \left.\left.-\varphi(s(\sigma))+\varphi\left(s\left(\sigma^{\prime}\right)\right)\right\}\right]\left[i k \left\{\frac{\partial \nu}{\partial n}-\frac{\partial \varphi}{\partial n}-\frac{1}{i k^{1 / 3}} R\left(k^{2 / 3} \zeta\right)\right.\right. \\
& \left.\times\left(\frac{\partial \widehat{\zeta}}{\partial n}-\frac{\partial \zeta}{\partial n}\right)\right\} \frac{1}{H\left(k^{2 / 3} \hat{\zeta}\right)}\left\{H\left(k^{2 / 3} \zeta\right) g_{0}+\frac{1}{i k^{1 / 3}} H^{\prime}\left(k^{2 / 3} \zeta\right) g_{1}\right\} \\
& +i k\left\{\zeta-\left(\frac{1}{i k^{1 / 3}} R\left(k^{2 / 3} \zeta\right)\right)^{2}\right\} \frac{\partial \zeta}{\partial n} g_{1}+\frac{1}{H\left(k^{2 / 3} \hat{\zeta}\right)}\left\{H\left(k^{2 / 3} \zeta\right) \frac{\partial g_{0}}{\partial n}\right. \\
& \left.\left.+\frac{1}{i k^{1 / 3}} H^{\prime}\left(k^{2 / 3} \zeta\right) \frac{\partial g_{1}}{\partial n}\right\}\right] \cdot v_{2}\left(k^{\varepsilon} \beta\right)^{2} k^{2} g\left(s\left(\sigma^{\prime}\right)\right) \\
& =\int_{I_{\beta}} d \beta \int_{\Sigma} d \eta \int_{I_{\sigma}} d \sigma^{\prime} \exp \left[i k \left\{\theta(s(\sigma), \eta, \beta)-\theta\left(s\left(\sigma^{\prime}\right), \eta, \beta\right)-\varphi(s(\sigma))\right.\right. \\
& \left.\left.\quad+\varphi\left(s\left(\sigma^{\prime}\right)\right)\right\}\right] a_{22}\left(s(\sigma), s\left(\sigma^{\prime}\right), \eta, \beta, p\right)(1+\alpha) k^{2} v_{2}\left(k^{\varepsilon} \beta\right)^{2} g\left(s\left(\sigma^{\prime}\right)\right)
\end{aligned}
$$

Posons

$$
\begin{aligned}
& a_{221}=i k\left\{\frac{\partial \nu}{\partial n}-\frac{\partial \varphi}{\partial n}+\frac{1}{i k^{1 / 3}} R\left(k^{2 / 3} \zeta\right)\left(\frac{\partial \zeta}{\partial n}-\frac{\partial \widehat{\zeta}}{\partial n}\right)\right\} \lambda(\sigma) \frac{D\left(\xi^{\prime}, \alpha\right)}{D(\eta, \beta)} \\
& a_{222}=i k\left\{\zeta-\left(\frac{1}{i k^{1 / 3}} R\left(k^{2 / 3} \zeta\right)\right)^{2}\right\} \frac{\partial \zeta}{\partial n} g_{1} \\
& a_{223}=a_{22}-a_{221}-a_{222},
\end{aligned}
$$

et

$$
\begin{aligned}
b_{22}= & \left\{k^{2 / 3} R\left(-\beta k^{2 / 3}\right)+\frac{1}{i} k^{1 / 3} \frac{2 \mu\left(1-\nabla \varphi(s) \cdot \nabla \theta_{0}(s, \eta)\right)}{\left(\nabla \rho_{0}(s, \eta)\right)^{2}}\right. \\
& \left.\times R^{\prime}\left(-\beta k^{2 / 3}\right)\right\} \frac{\partial \rho_{0}}{\partial n} \\
= & b_{221}+i b_{222} .
\end{aligned}
$$

Alors on a

$$
\begin{aligned}
& b_{221}=\left\{k^{2 / 3}\right. \operatorname{Re} R\left(-\beta k^{2 / 3}\right)+\mu \frac{2\left(1-\nabla \varphi(s) \cdot \nabla \theta_{0}(s, \eta)\right)}{\left(\nabla \rho_{0}(s, \eta)\right)^{2}} \\
&\left.\times \operatorname{Im} k^{1 / 3} R^{\prime}\left(-\beta k^{2 / 3}\right)\right\} \frac{\partial \rho_{0}}{\partial n}
\end{aligned}
$$

Grâce au comportement asymptotique de $R(z)$ nous avons deux lemmes suivants : 
Lemme 5.1. Pour une constante $C$ assez grande, on a

$$
\begin{aligned}
& -b_{221}(s, \eta, \beta, p) \geq c_{0}\left(-\frac{1}{\beta}+\frac{\mu}{\sqrt{-\beta}}\right) \quad \text { si } \quad-k^{2 / 3} \beta \geq C \\
& -b_{221}(s, \eta, \beta, p) \geq c_{0} k^{2 / 3} \quad \text { si }\left|\beta k^{2 / 3}\right| \leq C \\
& -b_{221}(s, \eta, \beta, p) \geq c_{0} k \sqrt{\beta} \quad \text { si } \beta k^{2 / 3} \geq C
\end{aligned}
$$

où $c_{0}$ est une constante positive.

Lemme 5. 2. On a pour tout $q=\left(q_{1}, q_{2}, q_{3}, q_{4}\right)$

$$
\begin{gathered}
\left|D_{s}^{q_{1}}\left(\frac{1}{k} \frac{\partial}{\partial \beta}\right)^{q_{2}}\left(\frac{1}{k} \frac{\partial}{\partial \eta}\right)^{q_{3}}\left(\frac{\partial}{\partial k}\right)^{q_{4}} b_{221}(s, \eta, \beta, k)\right| \\
\leq C_{q} k^{-\left(q_{2}+q_{8}+q_{4}\right) / 3}\left(-b_{221}(s, \eta, \beta, k)\right) .
\end{gathered}
$$

Comme nous avons démontré dans $\S 6$ de [4] il a lieu

$$
\left|i k\left\{\zeta-\left(\frac{1}{i k^{1 / 3}} R\left(k^{2 / 3} \zeta\right)\right)^{2}\right\} g_{1}\right| \leq C \frac{1}{\mu}\left(-b_{221}\right) .
$$

Et il est évident que

$$
a_{223} \in S_{1 / 3,0}^{0}\left(\Gamma_{0}\right)
$$

D'autre part

$$
\begin{aligned}
k^{2 / 3} R & \left(k^{2 / 3} \zeta\right) \frac{\partial \zeta}{\partial n}-b_{22}(s, \eta, \beta, p)-k^{2 / 3} R\left(-\beta k^{2 / 3}\right)\left(\frac{\partial \rho}{\partial n}-\frac{\partial \rho_{0}}{\partial n}\right) \\
= & {\left[k^{2 / 3} R\left(k^{2 / 3} \zeta\right)-\left\{k^{2 / 3} R\left(-\beta k^{2 / 3}\right)+\frac{1}{i} k^{1 / 3} \mu \frac{2\left(1-\nabla \varphi \cdot \nabla \theta_{0}\right)}{\left(\nabla \rho_{0}\right)^{2}}\right.\right.} \\
& \left.\left.\times R^{\prime}\left(-\beta k^{2 / 3}\right)\right\}\right] \frac{\partial \rho_{0}}{\partial n}+\left\{k^{2 / 3} R\left(k^{2 / 3} \zeta\right)-k^{2 / 3} R\left(-\beta k^{2 / 3}\right)\right\} \\
& \times\left(\frac{\partial \zeta}{\partial n}-\frac{\partial \rho_{0}}{\partial n}\right)+k^{2 / 3} R\left(-\beta k^{2 / 3}\right)\left(\frac{\partial \zeta}{\partial n}-\frac{\partial \rho}{\partial n}\right) .
\end{aligned}
$$

Selon les considération du $\S 6$ de [4] nous avons pour $|\beta| \leq k^{-\varepsilon}$

$$
\begin{aligned}
& \left|k^{2 / 3} R\left(k^{2 / 3} \zeta\right)-k^{2 / 3} R\left(-\beta k^{2 / 3}\right)-\frac{1}{i} k^{1 / 3} \cdot \mu \frac{2\left(1-\nabla \varphi \cdot \nabla \theta_{0}\right)}{\left(\nabla \rho_{0}\right)^{2}} R^{\prime}\left(-\beta k^{2 / 3}\right)\right| \\
& \quad \leq C \max \left(\frac{\mu}{k^{1 / 3}}\right)^{2}\left(-b_{221}(s, \eta, \beta, p)\right)
\end{aligned}
$$




$$
\begin{aligned}
& \quad \leq C a_{0}^{2}\left(-b_{221}(s, \eta, \beta, p)\right), \\
& \left|\left(k^{2 / 3} R\left(k^{2 / 3} \zeta\right)-k^{2 / 3} R\left(-\beta k^{2 / 3}\right)\right)\left(\frac{\partial \zeta}{\partial n}-\frac{\partial \rho_{0}}{\partial n}\right)\right| \\
& \quad \leq C k^{-\varepsilon}\left(-b_{221}(s, \eta, \beta, p)\right), \\
& \left|k^{2 / 3} R\left(-\beta k^{2 / 3}\right)\left(\frac{\partial \zeta}{\partial n}-\frac{\partial \rho}{\partial n}\right)\right| \leq C k^{-\varepsilon}\left(-b_{221}(s, \eta, \beta, p)\right)
\end{aligned}
$$

et

$$
\left|k^{2 / 3} R\left(-\beta k^{2 / 3}\right) \frac{\partial \widehat{\zeta}}{\partial n}\right| \leq C k^{-\varepsilon}\left(-b_{221}(s, \eta, \beta, p)\right)
$$

C'est ainsi que l'on a le

Lemme 5. 3. Pour $p=i k+\mu$ et $\beta$ tels que $0<\mu \leq a_{0} k^{1 / 3},|\beta| \leq k^{-\varepsilon}$ il a lieu

$$
\begin{gathered}
\mid a_{22}\left(s, s^{\prime}, \eta, \beta, p\right)-\left\{b_{22}(s, \eta, \beta, p)+k^{2 / 3} R\left(-\beta k^{2 / 3}\right)\left(\frac{\partial \rho}{\partial n}-\frac{\partial \rho_{0}}{\partial n}\right)\right. \\
\left.\quad+i k\left(\frac{\partial \nu}{\partial n}-\frac{\partial \varphi}{\partial n}\right)\right\} \frac{D\left(\xi^{\prime}, \alpha\right)}{D(\eta, \beta)} \mid \leq C a_{0}^{2}\left(-b_{221}\right) .
\end{gathered}
$$

En posant $\xi=k\left\{(1+\alpha) \xi^{\prime}-\varphi^{0}\right\}$ nous définissons $A_{22}, B_{22 j}$ etc. par

$$
\begin{aligned}
& A_{22}\left(s, \xi, s^{\prime}, p\right)= a_{22}\left(s, s^{\prime}, \eta\left(s, s^{\prime}, \xi^{\prime}, \alpha\right), \beta\left(s, s^{\prime}, \xi^{\prime}, \alpha\right), p\right) \\
& \times \frac{D\left(\xi^{\prime}, \alpha\right)}{D(\eta, \beta)}\left(s, s^{\prime}, \xi^{\prime}, \alpha\right) \\
& B_{22 j}\left(s, \xi, s^{\prime}, p\right)=b_{22 j}\left(s, s^{\prime}, \eta\left(s, s, \xi^{\prime}, \alpha\right), \beta\left(s, s^{\prime}, \xi^{\prime}, \alpha\right), p\right) B_{22}=B_{221}+i B_{222} \\
& \check{B}_{22 j}(s, \xi, p)=B_{22 j}(s, \xi, s, p), \\
& \check{B}_{22}=\dot{B}_{221}+i \check{B}_{222}, \\
& \cup_{2}\left(s, \xi, s^{\prime}, p\right)=v_{2}\left(k^{\varepsilon} \beta\left(s, s^{\prime}, \xi^{\prime}, \alpha\right)\right), \\
& \mathscr{A}_{22}=A_{22}\left(s, D_{s}, s^{\prime}, p\right) \\
& \mathscr{B}_{22 j}=B_{22 j}\left(s, D_{s}, s^{\prime}, p\right), \quad \mathscr{B}_{22}=\mathscr{B}_{221}+i \mathscr{B}_{222} \\
& \breve{\mathcal{B}}_{22 j}=\check{B}_{22 j}\left(s, D_{s}, p\right), \quad \breve{\mathcal{B}}_{22}=\breve{\mathscr{B}}_{221}+i \breve{\mathscr{B}}_{222}
\end{aligned}
$$




$$
\begin{aligned}
r_{2}=v_{2}\left(s, D_{s}, s^{\prime}, p\right) . \\
\frac{\left.\partial U_{22}\right|_{I}=\int_{I_{\beta}}}{\partial n} d \beta \int_{\Sigma} d \eta \int_{I_{\sigma}} d \sigma^{\prime} \cdot \exp \left[i k \left\{\theta(s(\sigma), \eta, \beta)-\theta\left(s\left(\sigma^{\prime}\right), \eta, \beta\right)\right.\right. \\
\left.\left.\quad-\varphi(s(\sigma))+\varphi\left(s\left(\sigma^{\prime}\right)\right)\right\}\right] \cdot a_{22}\left(s(\sigma), s\left(\sigma^{\prime}\right), \eta, \beta, p\right) \\
\quad \times(1+\alpha) k^{2} v_{2}\left(k^{\varepsilon} \beta\right)^{2} g\left(s\left(\sigma^{\prime}\right)\right) \\
=\int_{I_{\alpha}} d \alpha \int_{\Sigma} d \xi^{\prime} \int_{I_{\sigma}} d \sigma^{\prime} \exp \left[i k \left\{\left\langle\sigma-\sigma^{\prime}, \xi^{\prime}\right\rangle(1+\alpha)\right.\right. \\
\left.\left.\quad-\left\langle\sigma-\sigma^{\prime}, \varphi^{0}\right\rangle\right\}\right] A_{22}\left(s(\sigma), \xi, s\left(\sigma^{\prime}\right), p\right) v_{2}\left(s(\sigma), \xi, s\left(\sigma^{\prime}\right), p\right)^{2} \\
\quad \times(1+\alpha) k^{2} g\left(s\left(\sigma^{\prime}\right)\right) \\
=\int_{R^{2}} d \xi \int_{I_{\sigma}} d \sigma^{\prime} \exp \left\{i\left\langle\sigma-\sigma^{\prime}, \xi\right\rangle\right\} \\
\quad \times A_{22}\left(s(\sigma), \xi, s\left(\sigma^{\prime}\right), p\right) v_{2}\left(s(\sigma), \xi, s\left(\sigma^{\prime}\right) p\right)^{2} g\left(s\left(\sigma^{\prime}\right)\right) \\
=\left(A_{22} \circ \cup_{2}^{2}\right)\left(s(\sigma), D_{s}, s^{\prime}, p\right) g(s) .
\end{aligned}
$$

Posons

$$
\mathscr{Q}_{22}=\left(A_{22} \circ \cup_{2}^{2}\right)\left(s, D_{s}, s^{\prime}, p\right)-\Upsilon_{2}^{*} \mathcal{A}_{22} Y_{2}
$$

et nous avons

$$
\left.\frac{\partial U_{22}}{\partial n}\right|_{\Gamma}=\Upsilon_{2}^{*} \grave{A}_{22} \Upsilon_{2} g+\mathscr{P}_{22} g
$$

Grâce à la définition des $B_{22 j}$ et au comportement asymptotique de $R(z)$ nous avons le

Lemme 5.4. Pour $q=\left(q_{1}, q_{2}\right)$ tel que $\left|q_{2}\right| \geq 1$ il a lieu

$$
\left|D_{\sigma}^{q_{1}} \partial_{\xi}^{q_{2}} \check{B}_{222}(s(\sigma), \xi, p)\right| \leq C_{q} k^{-\left(\left|q_{2}\right|-1\right) / 3} \frac{1}{\mu}\left(-\breve{B}_{221}(s(\sigma), \xi, p)\right)
$$

Désignons par $\left[\check{B}_{22 j}\right]_{F}$ la partie de Friedrichs pour le symbole $\check{B}_{22 j}$ et nous considérons le symbole de $\breve{\mathscr{B}}_{222}-\left[\breve{B}_{222}\right]_{F}$ selon Kumano-go [6], le chapitre 3.

Symbole de $\left(\check{\mathscr{B}}_{222}-\left[\check{B}_{222}\right]_{F}\right)$

$$
\sim \sum_{|r|=1} \psi_{\gamma}(\xi) \check{B}_{222(\gamma)}(s, \xi, p)+\sum_{|r|+|\delta| \geq 2} \psi_{r, \delta}(\xi) \check{B}_{222(\gamma)}^{(r)}(s, \xi, p) .
$$


Puisque $\psi_{r}(\xi) \in S^{-1}$ nous avons

$$
\sum_{|r|=1} \psi_{r}(\xi) \breve{B}_{2222(r)}(s, \xi, p) \in S_{1 / 3,0}^{0}(\Gamma) .
$$

Notons que pour $|\gamma|+|\delta| \geq 2$

$$
\psi_{r, \delta}(\xi) \breve{B}_{222(\delta)}^{(r)} \in S_{1 / 3,0}^{1-1 / 3(|r|+|\delta|) / 2}(\Gamma) .
$$

Donc pour $\gamma, \delta$ tels que $|\gamma|+|\delta| \geq 6$ il a lieu

$$
\psi_{\gamma, \delta}(\xi) \check{B}_{222(\delta)}^{(Y)} \in S_{1 / 3,0}^{0}(\Gamma) \text {. }
$$

Soit $|\gamma| \geq 1$. D'après $\psi_{r, \delta}(\xi) \in S^{-(|r|-|\delta|) / 6}(\Gamma)$ et le lemme 5.4

$$
\begin{aligned}
\left|\psi_{r, \delta}(\xi) \dot{B}_{222(\delta)}^{(r)}(s, \xi, p)\right| & \leq C k^{(-1 / 3)(|r|-1)} k^{\left(|r|-\mid \delta^{i}\right) / 6} \frac{1}{\mu}\left(-B_{221}\right) \\
& \leq C k^{(2-|r|-|\delta|) / 6} \frac{1}{\mu}\left(-B_{221}\right) .
\end{aligned}
$$

Donc lorsque $|\gamma| \geq 1,|\gamma|+|\delta| \geq 2$ on a

$$
\left|\psi_{r, \delta}(\xi) \check{B}_{222(\delta)}^{(\gamma)}(s, \xi, p)\right| \leq \frac{C}{\mu}\left(-\check{B}_{221}\right) .
$$

Pour $|\delta|$ impaire $\geq 3$ d'après la définition de $\psi_{0, \delta}(\xi)$

$$
\psi_{0, \delta}(\xi) \in S_{1 / 3,0}^{-1-(|\delta|-1) / 6} .
$$

Donc pour $|\delta|$ impaire

$$
\psi_{0, \delta}(\xi) \check{B}_{222(\delta)} \in S_{1 / 3,0}^{-(|\delta|-1) / 6}(\Gamma) .
$$

Pour $|\delta|$ paire

$$
\phi_{0, \delta}(\xi) \breve{B}_{222(\delta)} \quad \text { est à valeurs réelles }
$$

et appartient à $S_{1 / 3,0}^{1-|\delta| / 6}(\Gamma)$. En appliquant le raisonnement jusqu’à maintenant nous avons

$$
\begin{aligned}
& \left|\psi_{0, \delta}(\xi) \breve{B}_{222(\delta)}(s, \xi, p)-\left[\psi_{0, \delta} \breve{B}_{222(\delta)}\right]_{F}-\sum_{\substack{\delta^{\prime} ;: \text { paire } \\
\mid \delta^{\prime}, \geq^{2}}} \psi_{0, \delta^{\prime}}(\xi) \psi_{0, \delta}(\xi) \breve{B}_{222\left(\delta+\delta^{\prime}\right)}\right| \\
& \leq C \frac{1}{\mu}\left(-\breve{B}_{221}\right) .
\end{aligned}
$$

D'autre part, $\psi_{0, \delta}(\xi) \psi_{0, \delta^{\prime}}(\xi) \check{B}_{222\left(\delta+\delta^{\prime}\right)}(s, \xi, p) \in S_{1 / 3,0}^{1-\left|\delta+\delta^{\prime}\right| / 6}(\Gamma)$. En répétant ce processus nous avons un symbole $\widetilde{B}(s, \xi, p)$ à valeurs réelles appartenant à $S_{1 / 3,0}^{1}(\Gamma)$ et $\widetilde{\mathscr{B}} \in S_{1 / 3,0}^{1}(\Gamma)$ tels que 


$$
\breve{\mathscr{B}}_{222}=\left[\breve{B}_{222}\right]_{F}+[\widetilde{B}]_{F}+\widetilde{\widetilde{B}}\left(s, D_{s}, p\right)
$$

et que

$$
|\widetilde{\mathscr{B}}(s, \xi, p)| \leq \frac{C}{\mu}\left(-\check{B}_{221}\right) \text {. }
$$

En posant $w_{2}=\Upsilon_{2} g$ nous avons

$$
\begin{aligned}
\left|\operatorname{Im}\left(\check{\mathscr{B}}_{222} w_{2}, w_{2}\right)_{m}\right| & \leq\left|\left(\widetilde{\mathscr{B}} w_{2}, w_{2}\right)_{m}\right| \\
& \leq \frac{C}{\mu}\left(\left[-\check{B}_{221}\right]_{F} w_{2}, w_{2}\right)_{m} .
\end{aligned}
$$

De cette façon on a la

\section{Proposition 5. 5.}

$$
\begin{aligned}
-\operatorname{Re} & \left(\mathcal{B}_{22} \Upsilon_{2} g, \Upsilon_{2} g\right)_{m} \\
& \geq\left(1-\frac{C}{\mu}-C k^{-\varepsilon / 2}\right)\left(\left[-B_{221}\right]_{F} \Upsilon_{2} g, \Upsilon_{2} g\right)_{m} .
\end{aligned}
$$

Et puis si l'on pose

$$
\begin{aligned}
& \mathscr{C}\left(s, \xi, s^{\prime}, p\right)=k^{2 / 3} R\left(\beta\left(s, s^{\prime}, \xi^{\prime}, \alpha\right) k^{2 / 3}\right)\left(\frac{\partial \rho}{\partial n}-\frac{\partial \rho_{0}}{\partial n}\right) \\
& \mathcal{E}\left(s, \xi, s^{\prime}, p\right)=i k\left(\frac{\partial \nu}{\partial n}\left(s, s^{\prime}, \eta, \beta, p\right)-\frac{\partial \varphi}{\partial n}(s)\right)
\end{aligned}
$$

on peut vérifier facilement qu'il a lieu des estimations

$$
\left|\operatorname{Re}\left(\mathscr{C}\left(s, D_{s}, s^{\prime}, p\right) w_{2}, w_{2}\right)_{m}\right| \leq C_{m} k^{-\varepsilon}\left(\left[-\check{B}_{221}\right]_{F} w_{2}, w_{2}\right)_{m}
$$

Du lemme 5.3 , de la proposition 5.5 , de $(5.6)$ et de (5.7) on déduit la

Proposition 5. 6. Il a lieu l'estimation

$$
\begin{aligned}
-\operatorname{Re}\left(\left.\frac{\partial U_{22}(p, g: x)}{\partial n}\right|_{\Gamma}, g\right)_{m} \\
\geq\left(1-C\left(\mu^{-1}+k^{-\varepsilon}+a_{0}^{2}\right)\right)\left(\left[-\dot{B}_{221}\right]_{F} \Upsilon_{2} g, \Upsilon_{2} g\right)_{m} \\
\quad-\operatorname{Re}\left(\mathcal{L}_{22} g, g\right)_{m}-C_{m}\|g\|_{m}^{2},
\end{aligned}
$$


pour toute $g(s) \in \mathscr{D}\left(\Gamma_{1}\right)$.

Corollaire. Soit $\gamma(s) \in \mathscr{D}\left(\Gamma_{1}\right)$. Pour toute $g(s) \in \mathscr{D}\left(\Gamma_{0}\right)$ il a lieu

$$
\begin{aligned}
& -\operatorname{Re}\left(\left.\frac{\partial U_{22}\left(p, \gamma(s)^{2} g(s): x\right)}{\partial n}\right|_{\Gamma}, g(s)\right)_{m} \\
& \quad \geq\left(1-C\left(\mu^{-1}+k^{-\varepsilon}+a_{0}^{2}\right)\right)\left(\left[-B_{221}\right]_{F} r_{2} \gamma g, \Upsilon_{2} \gamma g\right)_{m} \\
& -\operatorname{Re}\left(\mathscr{Q}_{22} \gamma g, \gamma g\right)_{m}-C k^{-\varepsilon / 2}\left(\left[-B_{221}\right]_{F} \tilde{\Upsilon}_{2} \widetilde{\gamma} g, \tilde{\Upsilon}_{2} \widetilde{\gamma} g\right)_{m}-C_{m}\|\gamma g\|_{m}^{2},
\end{aligned}
$$

ò̀ $\tilde{U}_{2}(l)=1$ pour $|l| \leq 3$ el $\widetilde{v}_{2}(l)=0$ pour $|l| \geq 4, \tilde{\Upsilon}_{2}$ est l'opérateur pseudo différential défini par le symbole $\widetilde{v}_{2}\left(k^{\varepsilon} \beta\left(s, s^{\prime}, \xi^{\prime}, \alpha\right)\right)$ et $\widetilde{\gamma}$ une fonction telle qu'elle est égale à 1 sur un voisinage du support de $\gamma(s)$.

Remarque. Nous avons fait une considération pour $p$ tel que $0<\mu \leq a_{0} k^{1 / 3}$, mais pour $k^{1 / 2} \geq \mu \geq a_{0} k^{1 / 3}$ nous pouvons achever le raisonnement en employant $\exp \left(\frac{2}{3} z^{3 / 2}\right)$ à la place de $H(z)$ et obtenir les estimations de la proposition 5.6 et de sa corollaire.

\section{$\S$ 6. Construction de $U_{21}$ et Ses Propriétés}

Afin de construire $U_{21}$ nous considérons un problème au bord (4.1) pour $\beta_{0} \geq-\beta \geq k^{-\varepsilon}, 0<\mu<k^{1 / 2}$. Cherchons $u$ sous la forme

$$
u(x, \eta, \beta, p)=\exp \{i k(\psi-\varphi)\} \cdot G .
$$

Puisque

$$
\begin{aligned}
\exp \{- & i k(\psi-\varphi)\} \cdot A_{\varphi} u \\
= & \left(1-(\nabla \varphi)^{2}\right) p^{2} G-2 p \nabla \varphi \cdot i k(\nabla \psi-\nabla \varphi) G-2 p \nabla \varphi \cdot \nabla G \\
& -(i k(\nabla \psi-\nabla \varphi))^{2} G-2 i k(\nabla \psi-\nabla \varphi) \cdot \nabla G-i k(\Delta \psi-\Delta \varphi) G \\
& -\Delta G-p \Delta \varphi \cdot G \\
= & \left\{p^{2}-(i k)^{2}\left(\nabla \psi+\frac{\mu}{i k} \nabla \varphi\right)^{2}\right\} \cdot G-2 i k\left(\nabla \psi+\frac{\mu}{i k} \nabla \varphi\right) \cdot \nabla G \\
& -i k\left(\Delta \psi+\frac{\mu}{i k} \Delta \varphi\right) G-\Delta G,
\end{aligned}
$$

nous choisissons $\psi$ de telle façon que 


$$
\left\{\begin{array}{l}
\left(\nabla \psi+\frac{\mu}{i k} \nabla \varphi\right)^{2}=\left(1+\frac{\mu}{i k}\right)^{2} \quad \text { dans } \bar{\Omega} \\
\phi=\theta(s, \eta, \beta) \quad \text { sur } \Gamma
\end{array}\right.
$$

et $G$ de telle façon que

(6. 3) $\left\{\begin{array}{l}2\left(\nabla \psi+\frac{\mu}{i k} \nabla \varphi\right) \cdot \nabla G+\left(\Delta \psi+\frac{\mu}{i k} \Delta \varphi\right) G+\frac{1}{i k} \Delta G=0 \quad \text { dans } \bar{\Omega} \\ G=v(s) \quad \text { sur } \quad \Gamma .\end{array}\right.$

D'abord considérons la construction de $\psi$ vérifiant (6.2).

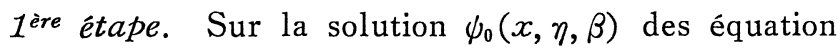

$$
\left\{\begin{array}{l}
\left(\nabla \psi_{0}\right)^{2}=1 \quad \text { dans } \bar{\Omega} \\
\psi_{0}(x, \eta, \beta)=\theta(x, \eta, \beta) \quad \text { sur } \quad \Gamma
\end{array}\right.
$$

nous avons les

Lemme 6.1. Une fonction $\psi(x, \eta, \beta)$ satisfaisant à (6.4) vérifie l'estimation

$$
\begin{gathered}
\left|\left(\frac{\partial}{\partial r}\right)^{q_{1}}\left(\frac{\partial}{\partial s}\right)^{q_{2}}\left(\frac{\partial}{\partial \sqrt{-\beta}}\right)^{q_{3}}\left(\frac{\partial}{\partial \eta}\right)^{q_{4}} \psi_{0}(x, \eta, \beta)\right| \\
\leq C_{q}(\sqrt{-\beta}+\sqrt{r})^{-\left(2 q_{1}+\left|q_{2}\right|+q_{3}+q_{4}\right)}
\end{gathered}
$$

Et plus pour $\psi_{0}$ telle que $\partial \psi_{0} / \partial r<0$ il a lieu

$$
\begin{aligned}
& -\left.\frac{\partial \psi_{0}}{\partial r}\right|_{\Gamma}=\sqrt{-\beta}\left|\nabla \rho_{0}(s, \eta)\right| \cdot\left(1+\beta c_{1}(s, \eta, \beta)\right) \\
& -\left.\Delta \psi_{0}\right|_{\Gamma}=\frac{1}{\sqrt{-\beta}} c_{2}(s, \eta, \beta)
\end{aligned}
$$

où $c_{j}(s, \eta, \beta) \in C^{\infty}\left(\Gamma \times \Sigma \times\left[-\beta_{0}, 0\right)\right)$ et $c_{2}(s, \eta, \beta) \geq c>0$.

Lemme 6.2. La solution de l'équation

$$
\left\{\begin{array}{l}
\nabla \psi_{0} \cdot \nabla f=h \quad \text { dans } \bar{\Omega} \\
\left.f\right|_{\Gamma}=v \quad \text { sur } \Gamma
\end{array}\right.
$$

pour les données $v(s) \in C\left(\Gamma_{0}\right), h(x) \in C^{\infty}\left(\bigcup_{\substack{s \in \Gamma_{0} \\ \eta \in \Sigma}} L^{+}(s, \eta)\right)$ admet l'estimation 


$$
\begin{gathered}
\left|\left(\frac{\partial}{\partial r}\right)^{q_{1}}\left(\frac{\partial}{\partial s}\right)^{q_{2}}\left(\frac{\partial}{\partial \beta}\right)^{q_{3}}\left(\frac{\partial}{\partial \eta}\right)^{q_{s}} f\right| \leq C_{q}\left\{\left(\frac{1}{\sqrt{-\beta}+\sqrt{r}}\right)^{5 \mid q !}|v|_{q}\right. \\
\left.+\sum_{j+l=|q|}\left(\frac{1}{\sqrt{-\beta}+\sqrt{r}}\right)^{5 j} \int_{s}^{x}|h|_{l} d r\right\} .
\end{gathered}
$$

Supposons que $\psi(x, \eta, \beta, p)$ vérifiant (6.2) se développe comme

$$
\psi(x, \eta, \beta, p)=\sum_{j=0}^{\infty} \psi_{j}(x, \eta, \beta)\left(\frac{\mu}{i k}\right)^{j} .
$$

En substituant $\psi$ à (6.2) et en égalant les coefficients de $\left(\frac{\mu}{i k}\right)^{j}$ nous avons

$$
\left(\nabla \psi_{0}\right)^{2}=1 \quad \text { dans } \quad \bar{\Omega}
$$

et pour $j \geq 1$

$$
\sum_{i=0}^{j} \nabla \psi_{j-l} \cdot \nabla \psi_{l}+2 \nabla \varphi \cdot \nabla \psi_{j-1}=N_{j}
$$

où $N_{1}=2, N_{2}=1$ et $N_{j}=0$ pour $j \geq 3$. Choissons $\psi_{0}$ de façon que $\left.\psi_{0}\right|_{r}$ $=\theta(s, \eta, \beta)$ et que $\partial \psi_{0} / \partial r<0$. Et puis nous déterminons les $\psi_{j}, j \geq 1$ de façon que $\psi_{j}=0$ sur $\Gamma$ et que (6.5) ait lieu. Sur $\psi_{1}$, par exemple, nous avons en utilisant le lemma 6.2

$$
\begin{aligned}
& \left|\left(\frac{\partial}{\partial r}\right)^{q_{1}}\left(\frac{\partial}{\partial s}\right)^{q_{2}}\left(\frac{\partial}{\partial \beta}\right)^{q_{3}}\left(\frac{\partial}{\partial \eta}\right)^{q_{4}} \psi_{1}\right| \\
& \quad \leq C_{q} \cdot \sum_{l+l^{\prime}=|q|}(\sqrt{-\beta}+\sqrt{r})^{-5 l} \int_{\delta_{0}}^{x}\left|\psi_{0}\right|_{l^{\prime}} d r
\end{aligned}
$$

d'après le lemme 6.1

$$
\begin{aligned}
& \leq C_{q} \cdot \sum_{l+l^{\prime}=|q|}(\sqrt{-\beta}+\sqrt{r})^{-5 l}(\sqrt{-\beta}+\sqrt{r})^{-5 l^{\prime}} \\
& \leq C_{q}(\sqrt{-\beta}+\sqrt{r})^{-5|q|} .
\end{aligned}
$$

De la même façon, à propos de $\psi_{2}$ nous avons

$$
\left|\left(\frac{\partial}{\partial r}\right)^{q_{1}}\left(\frac{\partial}{\partial s}\right)^{q_{2}}\left(\frac{\partial}{\partial \beta}\right)^{q_{3}}\left(\frac{\partial}{\partial \eta}\right)^{q_{4}} \psi_{2}\right| \leq C_{q^{2}}(\sqrt{-\beta}+\sqrt{r})^{-5|q|-5}
$$

et ainsi de suite en employant le lemme 6.2 pour $j \geq 2$

$$
\left|\left(\frac{\partial}{\partial r}\right)^{q_{1}}\left(\frac{\partial}{\partial s}\right)^{q_{2}}\left(\frac{\partial}{\partial \beta}\right)^{q_{3}}\left(\frac{\partial}{\partial \eta}\right)^{q_{4}} \psi_{j}\right| \leq C_{q j}(\sqrt{-\beta}+\sqrt{r})^{-5(|q|+j-1)}
$$




$$
\leq C_{q j}(\sqrt{-\beta})^{-5(|q|+j-1)}
$$

Donc pour $-\beta \geq k^{-\varepsilon}, 0<\varepsilon<\frac{1}{5} \times \frac{1}{2}$, l'estimation (6.6) nous permit de trouver une fonction $\phi(x, \eta, \beta, p)$ telle que

$$
\psi \sim \sum_{j=0}^{\infty} \psi_{j}(x, \eta, \beta)\left(\frac{\mu}{i k}\right)^{j} .
$$

Alors $\psi$ satisfait à

$$
\left(\nabla \psi+\frac{\mu}{i k} \nabla \varphi\right)^{2} \equiv\left(1+\frac{\mu}{i k}\right)^{2} \quad\left(\bmod k^{-\infty}\right) \quad \text { dans } \bar{\Omega}
$$

et à

$$
\left.\psi\right|_{\Gamma}=\theta(s, \eta, \beta) \quad \text { sur } \quad \Gamma .
$$

Rappelons que $\phi_{1}$ vérifie l'équation

$$
\nabla \psi_{0} \cdot \nabla \psi_{1}=1-\nabla \psi_{0} \cdot \nabla \varphi \geq 1-|\nabla \varphi|>c_{0}>0
$$

D'autre part nous avons choisi $\psi_{0}$ de façon que $\partial \psi_{0} / \partial r<0$. Donc il a lieu

$$
-\psi_{1}(x, \eta, \beta) \geq c(1-|\nabla \varphi|) r
$$

d'où on déduit

(6. 7) $|\exp \{i k(\phi(x, \eta, \beta, p)-\varphi(x))\}| \leq \exp \{-c(1-|\nabla \varphi|) \mu r\}$.

Passons à la construction de $G$ vérifiant (6.3). Supposons que $G$ se développe comme

$$
G=\sum_{j, l=0}^{\infty} G_{j l}(x, \eta, \beta) k^{-j}\left(\frac{\mu}{i k}\right)^{l} .
$$

En substituant $G$ en (6.3) et égalant les coefficients nous avons les relations auxquelles les $G_{j l}$ doivent satisfaire et nous pouvons déterminer les $G_{j l}(x, \eta, \beta)$ par récurrence. Et il a lieu l'estimation

$$
\begin{gathered}
\left|\left(\frac{\partial}{\partial r}\right)^{q_{1}}\left(\frac{\partial}{\partial s}\right)^{q_{2}}\left(\frac{\partial}{\partial \beta}\right)^{q_{3}}\left(\frac{\partial}{\partial \eta}\right)^{q_{s}} G_{j l}(x, \eta, \beta)\right| \\
\leq C_{q j l}(\sqrt{-\beta})^{-10(|q|+j+l)} \cdot \exp (c r) .
\end{gathered}
$$

L'estimation au-dessus permit de définir $G(x, \eta, \beta, p)$ par, si $0<\varepsilon<\frac{1}{10}$

$$
G \sim \sum_{j, l=0}^{\infty} G_{j l}(x, \eta, \beta) k^{-j}\left(\frac{\mu}{i k}\right)^{l} .
$$


Alors une fonction définie avec ces $\psi$ et $G$ par

$$
u=\exp \{i k(\psi-\varphi)\} G
$$

vérifie

(6. 8) $\quad\left|\partial_{x}^{r} A_{\varphi} u\right| \leq C_{N, r} k^{-N} \cdot \exp [-\{c(1-|\nabla \varphi|) \mu-C\} r], \quad \forall x \in \bar{\Omega}$

d'après (6.7) pour tous $\gamma$ et $N>0$, et évidement aussi

$$
\left.u\right|_{\Gamma}=\exp \{i k(\theta(s, \eta, \beta)-\varphi(s))\} \cdot v(s) .
$$

En utilisant la construction de solution asymptotique de (4.1) nous définissons $U_{21}$. Pour

$$
v(s)=\lambda(s) \chi_{2}\left(1+\alpha\left(s, s^{\prime}, \eta, \beta\right)\right)^{2}(1+\alpha) \frac{D\left(\xi^{\prime}, \alpha\right)}{D(\eta, \beta)}
$$

construisons $G\left(x, s^{\prime}, \eta, \beta, p\right)$ par la processus au-dessus. Posons

$$
\begin{gathered}
U_{21}(p, g: x)=\int_{I_{\beta}} d \beta \int_{\Sigma} d \eta \int_{I_{\sigma}} d \sigma^{\prime} \exp \left[i k \left\{\psi(x, \eta, \beta, p)-\theta\left(s\left(\sigma^{\prime}\right), \eta, \beta\right)\right.\right. \\
\left.\left.-\varphi(x)+\varphi\left(s\left(\sigma^{\prime}\right)\right)\right\}\right] G\left(x, s\left(\sigma^{\prime}\right), \eta, \beta, p\right) v_{1}\left(k^{\varepsilon} \beta\right)^{2} g\left(s\left(\sigma^{\prime}\right)\right) k^{2}
\end{gathered}
$$

Grâce à (6.8) et à (6.9) nous avons

$$
\begin{gathered}
\left\|A_{\varphi} U_{21}(p, g: x)\right\|_{m} \leq C_{N, m} k^{-N}\|g\|_{0} \\
\left.U_{21}(p, g: x)\right|_{\Gamma}=V_{21} g .
\end{gathered}
$$

Ensuite considérons $\left.\frac{\partial U_{21}}{\partial n}\right|_{\Gamma}$.

$$
\begin{aligned}
\left.\frac{\partial U_{21}}{\partial n}\right|_{\Gamma} & =\int_{I_{\beta}} d \beta \int_{\Sigma} d \eta \int_{I_{\sigma}} d \sigma^{\prime} \exp \left[i k \left\{0(s(\sigma), \eta, \beta)-\theta\left(s\left(\sigma^{\prime}\right), \eta, \beta\right)\right.\right. \\
- & \left.\left.\varphi(s(\sigma))+\varphi\left(s\left(\sigma^{\prime}\right)\right)\right\}\right]\left\{i k\left(\frac{\partial \psi}{\partial n}-\frac{\partial \varphi}{\partial n}\right) G+\frac{\partial G}{\partial n}\right\} v_{1}\left(k^{\varepsilon} \beta\right)^{2} g\left(s\left(\sigma^{\prime}\right)\right) k^{2} \\
& =\int_{I_{\beta}} d \beta \int_{\Sigma} d \eta \int_{I_{\sigma}} d \sigma^{\prime} \exp \left[i k \left\{\theta(s(\sigma), \eta, \beta)-\theta\left(s\left(\sigma^{\prime}\right), \eta, \beta\right)\right.\right. \\
& \left.\left.-\varphi(s(\sigma))+\varphi\left(s\left(\sigma^{\prime}\right)\right)\right\}\right] \cdot a_{21}\left(s(\sigma), s\left(\sigma^{\prime}\right), \eta, \beta, p\right) \\
& \times(1+\alpha) k^{2} v_{1}\left(k^{\varepsilon} \beta\right)^{2} g\left(s\left(\sigma^{\prime}\right)\right) .
\end{aligned}
$$

D'après le lemme 6.1

$$
\left.\frac{\partial \psi}{\partial n}\right|_{\Gamma}=-\sqrt{-\beta} \frac{1}{\left|\nabla \rho_{0}(s, \eta)\right|}(1+O(\beta))
$$




$$
-\frac{\mu}{i k} \frac{1-\nabla \psi_{0} \cdot \nabla \varphi}{\sqrt{-\beta}}\left|\nabla \rho_{0}(s, \eta)\right|\left(1+O\left(\frac{\mu}{k}+|\beta|\right)\right)
$$

et

$$
\begin{aligned}
\left.\frac{\partial G}{\partial n}\right|_{\Gamma} & =(-\Delta \psi+O(1)) \frac{1}{\frac{\partial \psi}{\partial n}} G+O\left(\frac{\mu}{k}\right) \\
& =\frac{1}{-\beta} c_{2}(s, \eta, \beta)\left(1+O\left(\sqrt{-\beta}+\frac{\mu}{k}\right)\right) \cdot G .
\end{aligned}
$$

Posons

$$
\begin{aligned}
b_{21}\left(s, s^{\prime}, \eta, \beta, p\right)= & -i k \sqrt{-\beta} \frac{1}{\left|\nabla \rho_{0}(s, \eta)\right|} \\
& -\frac{\mu}{\sqrt{-\beta}}\left(1-\nabla \psi_{0} \cdot \nabla \varphi\right)\left|\nabla \rho_{0}(s, \eta)\right|-\frac{1}{-\beta} c_{2}\left(s, s^{\prime}, \eta, \beta\right) \\
= & b_{211}+i b_{212} .
\end{aligned}
$$

Selon le paragraphe 5 définissons

$$
\begin{aligned}
& A_{21}, B_{21}, B_{21 j}, \breve{B}_{21}, \breve{B}_{21 j}, \\
& \mathscr{A}_{21}, \mathscr{B}_{21}, \mathscr{B}_{21 j}, \breve{\mathscr{B}}_{21}, \breve{\mathcal{B}}_{12 j}, \mathscr{L}_{21} .
\end{aligned}
$$

Alors par la même considération que $\S 5$ nous avons la

Proposition 6. 3. Pour toute $g(s) \in \mathscr{D}\left(\Gamma_{1}\right)$ il a lieu l'estimation

$$
\begin{aligned}
& -\operatorname{Re}\left(\left.\frac{\partial U_{21}(p, g: x)}{\partial n}\right|_{\Gamma}, g\right)_{m} \\
& \quad \geq\left(1-C\left(k^{-\varepsilon}+\frac{1}{\mu}\right)\right)\left(\left[-\check{B}_{211}\right]_{F} \Upsilon_{1} X_{2} g, \Upsilon_{1} X_{2} g\right)_{m} \\
& \quad-\operatorname{Re}\left(\mathscr{L}_{21} g, g\right)_{m}-C_{m}\|g\|_{m}^{2},
\end{aligned}
$$

où $X_{2}=\chi_{2}\left(\left|D_{s}\right| / k\right)$.

Corollaire. Soit $\gamma(s) \in \mathscr{D}\left(\Gamma_{1}\right)$. Alors pour toute $g(s) \in \mathscr{D}\left(\Gamma_{0}\right)$ nous avons

$$
-\operatorname{Re}\left(\left.\frac{\partial U_{21}\left(p, \gamma(s)^{2} g: x\right)}{\partial n}\right|_{\Gamma}, g\right)_{m}
$$




$$
\begin{aligned}
& \geq\left(1-C\left(k^{-\varepsilon}+\frac{1}{\mu}\right)\right)\left(\left[-\check{B}_{211}\right]_{F} \Upsilon_{1} X_{2} \gamma g, \Upsilon_{1} X_{2} \gamma g\right)_{m} \\
& -\operatorname{Re}\left(\mathscr{L}_{21} \gamma g, \gamma g\right)_{m}-C_{m}\|\gamma g\|_{m}^{2} \\
& -C \cdot k^{-\varepsilon / 2}\left(\left[-\check{B}_{211}\right]_{F} \widetilde{\Upsilon}_{1} \widetilde{X}_{2} \widetilde{\gamma} g, \Upsilon_{1} \widetilde{X}_{2} \widetilde{\gamma} g\right)_{m},
\end{aligned}
$$

où $\tilde{\gamma} \in \mathscr{D}\left(\Gamma_{1}\right)$ telle que $\gamma=1$ sur le support de $\gamma$, et

$$
\tilde{\chi}_{2}(l)=\left\{\begin{array}{ll}
1 & l<1-\alpha_{0} \\
0 & l>1-\alpha_{0} / 2
\end{array}, \widetilde{v}_{1}(l)= \begin{cases}1 & l<-1 \\
0 & l>-\frac{1}{2} .\end{cases}\right.
$$

\section{§ 7. Construction de $U_{23}$ et Ses Propriétés}

Considérons le problème (4.1) pour $p$ et $\beta$ tels que $0<\mu<k^{1 / 2}$, $\beta_{0} \geq \beta>k^{-\varepsilon}$. Nous construisons une solution asymptotique de (4.1) sous la forme

$$
u=\exp \{i k(\psi(x, \eta, \beta, p)-\varphi(x))\} \cdot G(x, \eta, \beta, p) .
$$

Premièrement cherchons une fonction vérifiant

$$
\left\{\begin{array}{l}
\left(\nabla \psi+\frac{\mu}{i k} \nabla \varphi\right)^{2} \equiv\left(1+\frac{\mu}{i k}\right)^{2}\left(\bmod \left(r+|\beta|+|p|^{-1}\right)^{\infty}\right) \\
\left.\psi\right|_{r}=\theta(s, \eta, \beta) .
\end{array}\right.
$$

La construction de telle fonction seras faite selon le processus dans $\S 8$ de [4], et on peut supposer que $\psi$ satisfasse à

$$
\operatorname{Im} \psi(x, \eta, \beta, p) \geq c \sqrt{\beta} r .
$$

Ensuite, la même considération permit de trouver une fonction $G$ telle que

$$
\left\{\begin{array}{l}
2\left(\nabla \psi+\frac{\mu}{i k} \nabla \varphi\right) \cdot \nabla G+\left(\Delta \psi+\frac{\mu}{i k} \Delta \varphi\right) G+\frac{1}{i k} \cdot \Delta G \equiv 0 \\
\quad\left(\bmod \left(r+|\beta|+\mid p_{i}^{-1}\right)^{\infty}\right) \\
\left.G\right|_{\Gamma}=v(s) .
\end{array}\right.
$$

Alors

$$
A_{\varphi} u=\exp \{i k(\psi-\varphi)\} \cdot\left[\left\{(i k+\mu)^{2}-(i k \nabla \psi+\mu \nabla \varphi)^{2}\right\} \cdot G\right.
$$




$$
\left.+i k\left\{2\left(\nabla \psi+\frac{\mu}{i k} \nabla \varphi\right) \cdot \nabla G+\left(\Delta \psi+\frac{\mu}{i k} \Delta \varphi\right) G\right\}+\Delta G\right] .
$$

D'après (7. 1) et (7.3)

$$
\begin{aligned}
&\left|A_{\varphi} u\right| \leq \exp \{i k(\psi-\varphi)\} \mid \cdot C_{N}\left(r+|\beta|+|p|^{-1}\right)^{N} \\
& \leq C_{N} \cdot \sum_{j=0}^{N}\left(\begin{array}{c}
N \\
j
\end{array}\right)\left(\beta+|p|^{-1}\right)^{N-j} \cdot \exp (-k \sqrt{\beta} r) \cdot r^{j} \\
& \leq C_{N} \cdot \sum_{j=0}^{N}\left(\begin{array}{c}
N \\
j
\end{array}\right)\left(\beta+|p|^{-1}\right)^{N-j}\left(\frac{k \sqrt{\beta}}{2}\right)^{-j} \\
& \quad \times \exp \left(-\frac{1}{2} k \sqrt{\beta} r\right) \sup _{r \geq 0}\left(\exp (-r) \cdot r^{j}\right) .
\end{aligned}
$$

Puisqu'il a lieu par la supposition $\operatorname{sur} \beta$

$$
k \sqrt{\beta} \geq k^{1 / 2},
$$

nous avons

$$
\left|A_{\varphi} u\right| \leq C_{N} \cdot|p|^{-N} \exp \left(-\frac{1}{2} k^{1 / 2} \cdot r\right) .
$$

Prenons $G\left(x, s^{\prime}, \eta, \beta, p\right)$ comme une fonction vérifiant (7.3) en posant

$$
v(s)=\chi_{2}\left(1+\alpha\left(s, s^{\prime}, \eta, \beta\right)\right)^{2} \frac{D\left(\xi^{\prime}, \alpha\right)}{D(\eta, \beta)} \lambda(s)
$$

et définissons $U_{23}(p, g: x)$ par

$$
\begin{aligned}
U_{23}(p, g: x)= & \int_{I_{\beta}} d \beta \int_{\Sigma} d \eta \int_{I_{\sigma}} d \sigma^{\prime} \cdot \exp [i k\{\psi(x, \eta, \beta, p) \\
& \left.\left.-\theta\left(s\left(\sigma^{\prime}\right), \eta, \beta\right)-\varphi(x)+\varphi\left(s\left(\sigma^{\prime}\right)\right)\right\}\right] \\
& \times G\left(x, s\left(\sigma^{\prime}\right), \eta, \beta, p\right) \cdot v_{3}\left(k^{\varepsilon} \beta\right)^{2} g\left(s\left(\sigma^{\prime}\right)\right) k^{2} .
\end{aligned}
$$

Grâce à (7.1) et à (7.4) nous avons

$$
\begin{aligned}
& \left\{\begin{array}{l}
\left\|A_{\varphi} U_{23}\right\|_{m} \leq C_{N, m}|p|^{-N}\|g\|_{0} \\
\left.U_{23}\right|_{\Gamma}=V_{23} g .
\end{array}\right. \\
\left.\frac{\partial U_{23}}{\partial n}\right|_{\Gamma}= & \int_{I_{\beta}} d \beta \int_{\Sigma} d \eta \int_{I_{\sigma}} d \sigma^{\prime} \cdot \exp [i k\{\theta(s(\sigma), \eta, \beta) \\
& \left.\left.-\theta\left(s\left(\sigma^{\prime}\right), \eta, \beta\right)-\varphi(s(\sigma))+\varphi\left(s\left(\sigma^{\prime}\right)\right)\right\}\right] \\
& \times\left\{i k\left(\frac{\partial \psi}{\partial n}-\frac{\partial \varphi}{\partial n}\right) G+\frac{\partial G}{\partial n}\right\} v_{3}\left(k^{\varepsilon} \beta\right)^{2} k^{2} g\left(s\left(\sigma^{\prime}\right)\right)
\end{aligned}
$$




$$
\begin{aligned}
= & \int_{I_{\beta}} d \beta \int_{\Sigma} d \eta \int_{I_{\sigma}} d \sigma^{\prime} \cdot \exp \left\{i k\left\langle\sigma-\sigma^{\prime}, \xi^{\prime}\right\rangle(1+\alpha)-\left\langle\sigma-\sigma^{\prime}, \varphi^{0}\right\rangle\right\} \\
& \times a_{23}\left(s(\sigma), s\left(\sigma^{\prime}\right), \eta, \beta, p\right) k^{2} g\left(s\left(\sigma^{\prime}\right)\right)
\end{aligned}
$$

Posons

$$
b_{23}\left(s, s^{\prime}, \xi, p\right)=-k \sqrt{\beta\left(s, s^{\prime}, \xi^{\prime}, \alpha\right)} .
$$

Alors nous avons la

Proposition 7.1. Pour toute $g(s) \in \mathscr{D}\left(\Gamma_{1}\right)$ il a lieu l'estimation

$$
\begin{gathered}
-\operatorname{Re}\left(\left.\frac{\partial U_{23}}{\partial n}\right|_{\Gamma}, g\right)_{m} \geq\left(\left[-\check{B}_{23}\right]_{F} \Upsilon_{3} X_{2} g, \Upsilon_{3} X_{2} g\right)_{m} \\
-\operatorname{Re}\left(\mathscr{L}_{23} g, g\right)_{m}-C_{m}\|g\|_{m}^{2} .
\end{gathered}
$$

Corollaire. Soit $\gamma(s) \in \mathscr{D}\left(\Gamma_{1}\right)$. Alors pour toute $g \in \mathscr{D}\left(\Gamma_{0}\right)$ nous avons

$$
\begin{gathered}
-\operatorname{Re}\left(\left.\frac{\partial U_{23}\left(p, \gamma^{2} g: x\right)}{\partial n}\right|_{\Gamma}, g\right)_{m} \geq\left(\left[-\breve{B}_{23}\right]_{F} \Upsilon_{3} X_{2} \gamma g, \Upsilon_{3} X_{2} \gamma g\right)_{m} \\
-\operatorname{Re}\left(\mathscr{P}_{23} \gamma g, \gamma g\right)_{m}-C\|\gamma g\|_{m}^{2} \\
-C \cdot k^{-\varepsilon / 2}\left(\left[-\breve{B}_{23}\right]_{F} \tilde{\Upsilon}_{3} \widetilde{X}_{2} \widetilde{\gamma} g, \widetilde{\Upsilon}_{3} \widetilde{X}_{2} \widetilde{\gamma} g\right)_{m} .
\end{gathered}
$$

§ 8. Démonstration du Théorème 2. 1

Posons

$$
U_{2}(p, g: x)=\sum_{j=1}^{3} U_{2 j}(p, g: x)
$$

pour $g \in \mathscr{D}\left(\Gamma_{1}\right)$. D'après les résultats des paragraphes $4 \sim 7$ il a lieu

$$
\left\{\begin{array}{l}
\left\|A_{\varphi} U_{2}\right\|_{m} \leq C_{N, m}|p|^{-N}\|g\|_{0} \\
\left\|\left.U_{2}\right|_{\Gamma}-V_{2} g\right\|_{m} \leq C_{N, m}|p|^{-N}\|g\|_{0} .
\end{array}\right.
$$

Et puis nous avons

$$
-\operatorname{Re}\left(\left.\frac{\partial U_{2}(p, g: x)}{\partial n}\right|_{\Gamma}, g\right)_{m} \geq \frac{1}{2}\left(B_{2 F} X_{2} g, X_{2} g\right)_{m}-C_{m}\|g\|_{m}^{2}
$$

où 


$$
B_{2 F}=\sum_{j=1}^{3} Y_{j}^{*}\left[-\check{B}_{2 j}\right]_{F} Y_{j}
$$

puisqu'il a lieu

$$
\left|\sum_{j=1}^{3}\left(\mathscr{Q}_{2 j} g, g\right)_{m}\right| \leq C \cdot k^{-\varepsilon / 2}\left(B_{2 F} X_{2} g, X_{2} g\right)_{m}+C\|g\|_{m}^{2} .
$$

Et aussi nous avons

$$
\begin{gathered}
-\operatorname{Re}\left(\left.\frac{\partial U_{2}\left(p, \gamma^{2} g: x\right)}{\partial n}\right|_{\Gamma}, g\right)_{m} \geq\left(B_{2 F} X_{2} \gamma g, X_{2} \gamma g\right)_{m} \\
-C\|\gamma g\|_{m}^{2}-k^{-\varepsilon / 2}\left(B_{2 F} \widetilde{X}_{2} \widetilde{\gamma} g, \widetilde{X}_{2} \widetilde{\gamma}^{g}\right)_{m}
\end{gathered}
$$

pour $\gamma \in \mathscr{D}\left(\Gamma_{1}\right)$ et $g \in \mathscr{D}\left(\Gamma_{0}\right)$.

Puis construisons $U_{1}$ et $U_{3}$.

Pour $\eta \in\left\{\eta \in \boldsymbol{R}^{2} ;|\eta| \leq 1-\alpha_{0} / 2\right\}$ nous construisons une fonction $\theta_{0}(s, \eta) \in C^{\infty}\left(\Gamma_{0}\right)$ en suivant la considération dans $\S 3$ telle que

$$
\left\{\begin{array}{l}
\left|\nabla \theta_{0}(s, \eta)\right|^{2}=|\eta|^{2} \\
\frac{\partial \theta_{0}}{\partial \sigma_{j}}(0, \eta)=\eta_{j}, \quad j=1,2 .
\end{array}\right.
$$

Alors on peut trouver une fonction ${\psi_{0}}^{+}(x, \eta) \in C^{\infty}(\Gamma \times[0, \infty))$ telle que

$$
\begin{array}{ll}
\left|\nabla \psi_{0}^{+}(x, \eta)\right|^{2}=1 & \text { dans } \bar{\Omega} \\
\phi_{0}^{+}(x, \eta)=\theta_{0}(s, \eta) \quad \text { sur } \quad \Gamma_{0} \\
\frac{\partial \psi_{0}^{+}}{\partial n}<0 .
\end{array}
$$

D'autre part la méthode de construction de $\theta_{0}(s, \eta)$ assure l'existence d'une fonction indéfiniment différentiable $\eta\left(s, s^{\prime}, \xi\right)$ pour $|\xi| \leq 1-\alpha_{0}$ vérifiant

$$
\begin{gathered}
\theta_{0}(s(\sigma), \eta)-\theta_{0}\left(s\left(\sigma^{\prime}\right), \eta\right)-\varphi(s(\sigma))+\varphi\left(s\left(\sigma^{\prime}\right)\right) \\
=\left\langle\sigma-\sigma^{\prime}, \xi\right\rangle-\left\langle\sigma-\sigma^{\prime}, \varphi^{0}\right\rangle \\
\eta(0,0, \xi)=\xi .
\end{gathered}
$$

Alors $V_{1} g$ s'exprime

$$
V_{1} g(s(\sigma))=\lambda(s(\sigma)) \int_{\boldsymbol{R}^{2}} d \eta \int_{I_{\sigma}} d \sigma^{\prime} \exp \left\{i k \left(\theta_{0}(s(\sigma), \eta)-\theta_{0}\left(s\left(\sigma^{\prime}\right), \eta\right)\right.\right.
$$




$$
\begin{aligned}
& \left.\left.-\varphi(s(\sigma))+\varphi\left(s\left(\sigma^{\prime}\right)\right)\right)\right\} \cdot \chi_{1}\left(\left|\xi\left(s(\sigma), s\left(\sigma^{\prime}\right), \eta\right)\right|\right)^{2} \\
& \times \frac{\partial \xi}{\partial \eta} k^{2} g\left(s\left(\sigma^{\prime}\right)\right) .
\end{aligned}
$$

En utilisant le raisonnement de $\S 4$ nous pouvons définir $U_{1}$ vérifiant

$$
\left\{\begin{array}{l}
\left\|A_{\varphi} U_{1}\right\|_{m} \leq C_{N, m}|p|^{-N}\|g\|_{0} \\
\left.U_{1}\right|_{\Gamma}=V_{1} g
\end{array}\right.
$$

par

$$
\begin{aligned}
U_{1}(p, g: x)= & \int_{\boldsymbol{R}^{2}} d \eta \int_{I_{\sigma}} d \sigma^{\prime} \exp \left[i k \left\{\psi(x, \eta, p)-\varphi(x)-\theta_{0}\left(s\left(\sigma^{\prime}\right), \eta\right)\right.\right. \\
& \left.\left.+\varphi\left(s\left(\sigma^{\prime}\right)\right)\right\}\right] G\left(x, s\left(\sigma^{\prime}\right), \eta, p\right) k^{2} g\left(s\left(\sigma^{\prime}\right)\right)
\end{aligned}
$$

où $\psi(x, \eta, p)$ est une fonction vérifiant

$$
\left\{\begin{array}{l}
\left(\nabla \psi(x, \eta, p)+\frac{\mu}{i k} \nabla \varphi(x)\right)^{2} \equiv\left(1+\frac{\mu}{i k}\right)^{2} \quad\left(\bmod |p|^{-\infty}\right) \quad \text { dans } \bar{\Omega} \\
\psi(x, \eta, p)=\theta_{0}(s, \eta) \quad \text { sur } \Gamma
\end{array}\right.
$$

et $G\left(x, s^{\prime}, \eta, p\right)$ vérifie

$$
\left\{\begin{array}{c}
2\left(\nabla \psi+\frac{\mu}{i k} \nabla \varphi\right) \nabla G+\left(\Delta \psi+\frac{\mu}{i k} \Delta \varphi\right) G+\frac{1}{i k} \Delta G \equiv 0 \\
\left(\bmod |p|^{-\infty}\right) \quad \text { dans } \bar{\Omega} \\
G=\lambda(s(\sigma)) \chi_{1}\left(\left|\xi\left(s, s^{\prime}, \eta\right)\right|\right)^{2} \frac{\partial \xi}{\partial \eta} \quad \text { sur } \quad \Gamma .
\end{array}\right.
$$

Alors

$$
\begin{array}{r}
\left.\frac{\partial U_{1}(p, g: x)}{\partial n}\right|_{\Gamma}=\int_{R^{2}} d \eta \int_{I_{\sigma}} d \sigma^{\prime} \cdot \exp \left\{i k \left(\theta_{0}(s(\sigma), \eta)-\theta_{0}\left(s\left(\sigma^{\prime}\right), \eta\right)\right.\right. \\
\left.\left.-\varphi(s(\sigma))+\varphi\left(s\left(\sigma^{\prime}\right)\right)\right)\right\} \cdot\left\{i k\left(\frac{\partial \psi}{\partial n}-\frac{\partial \varphi}{\partial n}\right) G+\frac{\partial G}{\partial n}\right\} k^{2} g\left(s\left(\sigma^{\prime}\right)\right),
\end{array}
$$

et on a

$$
i k\left(\frac{\partial \psi}{\partial n}-\frac{\partial \varphi}{\partial n}\right)=i k\left\{-\sqrt{1-\eta^{2}}-\frac{\partial \varphi}{\partial n}\right\}+\mu \frac{1-\nabla \psi_{0} \cdot \nabla \varphi}{\sqrt{1-\eta^{2}}}+O(1) .
$$

En posant

$$
b_{1}=i k\left\{-\sqrt{1-\eta^{2}}-\frac{\partial \varphi}{\partial n}\right\}+\mu \frac{1-\nabla \psi_{0} \cdot \nabla \varphi}{\sqrt{1-\eta^{2}}}
$$




$$
=b_{11}+i b_{12}
$$

nous avons pour $g \in \mathscr{D}\left(\Gamma_{1}\right)$

$$
-\operatorname{Re}\left(\left.\frac{\partial U_{1}(p, g: x)}{\partial n}\right|_{\Gamma}, g\right)_{m} \geq\left(\left[-\breve{B}_{11}\right]_{F} X_{1} g, X_{1} g\right)_{m}-C\|g\|_{m}^{2} .
$$

Et aussi pour $\gamma \in \mathscr{D}\left(\Gamma_{1}\right), g \in \mathscr{D}\left(\Gamma_{0}\right)$

$$
-\operatorname{Re}\left(\left.\frac{\partial U_{1}\left(p, \gamma^{2} g: x\right)}{\partial n}\right|_{\Gamma}, g\right)_{m} \geq\left(\left[-\breve{B}_{11}\right]_{F} X_{1} \gamma g, X_{1} \gamma g\right)_{m}-C\|\widetilde{\gamma} g\|_{m}^{2} .
$$

Concernant $U_{3}$, on peut construire $U_{3}(p, g: x)$ vérifiant

$$
\left\{\begin{array}{l}
\left.\left|\left\|A_{\varphi} U_{3}\right\|_{m} \leq C_{N, m}\right| p\right|^{-N}\|g\|_{0} \\
\left.U_{3}\right|_{\Gamma}=V_{3} g
\end{array}\right.
$$

en utilisant le raisonnement de $\S 7$. Alors nous avons pour $g \in \mathscr{D}\left(\Gamma_{1}\right)$

$$
-\operatorname{Re}\left(\left.\frac{\partial U_{3}(p, g: x)}{\partial n}\right|_{\Gamma}, g\right)_{m} \geq c k\left\|X_{3} g\right\|_{m}^{2}-C \cdot\|g\|_{m}^{2}
$$

et aussi pour $\gamma \in \mathscr{D}\left(\Gamma_{1}\right), g \in \mathscr{D}\left(\Gamma_{0}\right)$

$$
-\operatorname{Re}\left(\left.\frac{\partial U_{3}\left(p, \gamma^{2} g: x\right)}{\partial n}\right|_{\Gamma}, g\right)_{m} \geq c k\left\|X_{3} \gamma g\right\|_{m}^{2}-C\|\widetilde{\gamma} g\|_{m}^{2}
$$

Alors pour $g \in \mathscr{D}\left(\Gamma_{1}\right)$ définissons $U(p, g: x)$ par

$$
U(p, g: x)=\sum_{j=1}^{3} U_{j}(p, g: x) \text {. }
$$

Il est évident qu'il a lieu d'après (8.1), (8.4), (8.7)

$$
\begin{aligned}
& \left\|A_{\varphi} U(p, g: x)\right\|_{m} \leq C_{N, m}|p|^{-N}\|g\|_{0} \\
& \left\|\left.U(p, g: x)\right|_{\Gamma}-g\right\|_{m} \leq C_{N, m}|p|^{-N}\|g\|_{0} .
\end{aligned}
$$

Posons

$$
B_{F}=X_{1}^{*} B_{2 F} X_{2}+X_{2}^{*}\left[-\check{B}_{11}\right]_{F} X_{1}+c k X_{3}^{*} X_{3}
$$

et nous avons pour $g \in \mathscr{D}\left(\Gamma_{1}\right)$

$$
-\operatorname{Re}\left(\left.\frac{\partial U(p, g: x)}{\partial n}\right|_{\Gamma}, g\right)_{m} \geq\left(B_{F} g, g\right)_{m}-C_{m}\|g\|_{m}^{2}
$$

Et aussi nous avons

(8. 12) $-\operatorname{Re}\left(\left.\frac{\partial U\left(p, \gamma^{2} g: x\right)}{\partial n}\right|_{\Gamma}, g\right)_{m} \geq\left(B_{F} \gamma g, \gamma g\right)_{m}-C_{m}\|g\|_{m}^{2}$. 
La définition de $U(p, g: x)$ pour $g \in C^{\infty}(\Gamma)$ sera faite comme suit: Prenons $\gamma_{j}(s) \in C^{\infty}(\Gamma), j=1,2, \cdots, J$, de telle façon que

$$
\sum_{j=1}^{J} \gamma_{j}(s)^{2}=1 \quad \text { sur } \quad \Gamma,
$$

et que le support de chaque $\gamma_{j}$ soit si petit qu'on puisse faire la construction de $U$ dans un voisinage du support. Et pour $g \in C^{\infty}(\Gamma)$ posons

$$
U(p, g: x)=\sum_{j=1}^{J} U\left(p, \gamma_{j}^{2} g: x\right) \text {. }
$$

Alors par les considérations jusqu'à maintenant on a

$$
\begin{aligned}
& \left\|A_{\varphi} U(p, g: x)\right\|_{m} \leq C_{N, m}|p|^{-N}\|g\|_{0} \\
& \left\|\left.U(p, g: x)\right|_{\Gamma}-g\right\|_{m} \leq C_{N, m}|p|^{-N}\|g\|_{0} .
\end{aligned}
$$

Et

$$
\begin{aligned}
-\operatorname{Re} & \left(\left.\frac{\partial U(p, g: x)}{\partial n}\right|_{\Gamma}, g\right)_{m} \\
& =\sum_{j=1}^{J}\left\{-\operatorname{Re}\left(\left.\frac{\partial U\left(p, \gamma_{j}^{2} g: x\right)}{\partial n}\right|_{\Gamma}, g\right)_{m}\right\} \\
& \geq \sum_{j=1}^{J} \mu \cdot \inf \frac{\left(1-\nabla \psi_{0}^{+} \cdot \nabla \varphi\right)}{\sqrt{1-\eta^{2}}}\left\|\gamma_{j} g\right\|_{m}^{2}-C_{m}\|g\|_{m}^{2} \\
& \geq \mu \cdot \inf \frac{\left(1-\nabla \psi_{0}^{+} \cdot \nabla \varphi\right)}{\sqrt{1-\eta^{2}}}\|g\|_{m}^{2}-C_{m}\|g\|_{m}^{2},
\end{aligned}
$$

puisqu'il a lieu

$$
\begin{aligned}
& X_{1}^{*}\left[\check{B}_{11}\right]_{F} X_{1} \geq \mu \cdot X_{1}^{*}\left(\inf _{|\eta| \leq \alpha_{0}} \frac{\left(1-\nabla \psi_{0}^{+} \cdot \nabla \varphi\right)}{\sqrt{1-\eta^{2}}}\right) X_{1} \\
& X_{2}^{*} B_{2 F} X_{2} \geq \frac{\mu}{2 \sqrt{\alpha_{0}}} X_{2}^{*} X_{2} .
\end{aligned}
$$

En tenant compte que $B_{\varphi}$ s'exprime comme

$$
B_{\varphi}(p, \partial / \partial x)=\frac{\partial}{\partial n}+v \frac{\partial}{\partial s}+p(v \cdot \nabla \varphi+c)+d
$$

où $v \frac{\partial}{\partial s}$ est un champ de vecteur réel sur $\Gamma$,

$$
-\operatorname{Re}\left(B_{\varphi} U(p, g: x), g\right)_{m} \geq\left(B_{F} g, g\right)_{m}-\mu((b \cdot \nabla \varphi+c) g, g)_{m}-C_{m}\|g\|_{m}^{2}
$$




$$
=\left(\left(B_{F}-\mu(b \cdot \nabla \varphi+c)\right) g, g\right)_{m}-C_{m}\|g\|_{m}^{2} \text {. }
$$

Notons qu'il a lieu l'estimation

$$
\begin{aligned}
& B_{F}-\mu \cdot(b \cdot \nabla \varphi+c) \\
& \geq \mu \cdot \inf _{\substack{|\eta|<1 \\
x \in \Gamma}}\left\{\left(\frac{1-\nabla \psi_{0}^{+}(x, \eta) \cdot \nabla \varphi(x)}{\sqrt{1-\eta^{2}}}\right)-b \cdot \nabla \varphi-c\right\} \\
& \geq \mu \cdot \inf _{\substack{|\eta|<1 \\
x \in \Gamma}}\left\{\frac{1-\eta \cdot \varphi_{s}}{\sqrt{1-\eta^{2}}}-v \cdot\left|\varphi_{s}\right|-c\right\} \\
& \geq \mu \cdot \inf _{x \in \Gamma}\left\{\sqrt{1-\left|\varphi_{s}\right|^{2}}-v \cdot\left|\varphi_{s}\right|-c\right\} .
\end{aligned}
$$

Admettons le

\section{Lemme 8. 1 .}

$$
c(\varphi)=\inf _{x \in \Gamma}\left(\sqrt{1-\left|\varphi_{s}\right|^{2}}-v \cdot\left|\varphi_{s}\right|-c\right)
$$

est positif lorsque

$$
\sup \left|\varphi_{s}\right|<\inf _{s \in \Gamma} \frac{-c(s) v(s)+\sqrt{1+\left|v(s)^{2}-c(s)^{2}\right|}}{1+v(s)^{2}} .
$$

Donc il a lieu pour toute $g \in C^{\infty}(\Gamma)$

$$
-\operatorname{Re}\left(B_{\varphi}(p, \partial / \partial x) U(p, g: x), g\right)_{m} \geq\left(c_{0}(\varphi) \mu-C_{m}\right)\|g\|_{m}^{2} 。
$$

C'est ainsi que nous avons démontré le théorème 2.1 au cas où $0<\mu<k^{1 / 2}$. Nous omettons la démonstration du théorème 2.1 pour $p$ tel que $|k|^{1 / 2} \leq \mu$.

\section{§ 9. La Nécessité de la Condition (1.3) et sur la Vitesse Propagatrice}

Pour démontrer la nécessité de la condition (1.3), nous n'avons qu'à vérifier que le théorème de Kajitani [5] est applicable. Mais afin d'appliquer sa méthode pour la considération de la vitesse propagatrice nous donnons d'abord une démonstration de la nécessité de (1.3) selon la méthode de Kajitani.

Supposons que $c\left(s_{0}\right) \geq 1$ pour $s_{0} \in \Gamma$ et que le problème mixte $(\mathrm{P})$ 
soit bien posé au sense de $C^{\infty}$ et ait la vitesse finie de propagation. Soit $U$ un voisinage dans $\boldsymbol{R}^{3}$ de $s_{0} . \Psi$ est une transformation définie dans $U$ telle que

$$
\begin{aligned}
& \Psi\left(s_{0}\right)=0 \\
& \Omega \cap U \rightarrow \boldsymbol{R}_{+}^{3}=\left\{\left(x_{1}, x_{2}, x_{3}\right) ; x_{3}>0\right\} \\
& \Gamma \cap U \rightarrow\left\{x ; x_{3}=0\right\} .
\end{aligned}
$$

Désignons par $A(x, \partial / \partial x, \partial / \partial t)$ et $\widetilde{B}(x, \partial / \partial x, \partial / \partial t)$ les opérateurs transformés par $\Psi$ de $\square$ et de $B$. On déduit de la supposition sur (P) que le problème mixte pour $(A, \widetilde{B})$ dans $\boldsymbol{R}_{+}^{3}$ est locallement solvable dans un voisinage de l'origine et qu'il a une vitesse propagatrice $v_{0}$. Posons

$$
\begin{aligned}
& \Lambda\left(x_{0}, t_{0}\right)=\left\{(x, t) ;\left|x-x_{0}\right| \leq v_{0}\left(t_{0}-t\right), 0 \leq t \leq t_{0}, x_{3} \geq 0\right\} \\
& D\left(x_{0}, t_{0}\right)=\left\{(x, 0) ;\left|x-x_{0}\right| \leq v_{0} t_{0}, x_{3} \geq 0\right\} \\
& G\left(x_{0}, t_{0}\right)=\left\{(x, t) ;(x, t) \in \Lambda\left(x_{0}, t_{0}\right), x_{3}=0\right\} .
\end{aligned}
$$

D'après la supposition et l'appendice de Mizohata [10], il a lieu que pour tout $m \geq 0$ et pour tout $(x, t) \in \Psi(\bar{\Omega} \cap U) \times\{t>0\}$

$$
|u|_{m, A(x, t)} \leq C_{m}\left\{|A u|_{m^{\prime}, A(x, t)}+|\widetilde{B} u|_{m^{\prime}, G(x, t)}+|u|_{m^{\prime}, D(x, t)}\right\}
$$

où $|u|_{m, \omega}=\sup _{\substack{x \in \omega \\|a| \leq m}}\left|D^{\alpha} u\right|$ et $m^{\prime}$ est une constante dépende de $m$ et indépendente de $(x, t)$.

Posons

$$
\begin{aligned}
& A(n)=A\left(\frac{x}{n}, n \frac{\partial}{\partial x}, n \frac{\partial}{\partial t}\right) \\
& B(n)=\widetilde{B}\left(\frac{x}{n}, n \frac{\partial}{\partial x}, n \frac{\partial}{\partial t}\right), n=1,2, \cdots
\end{aligned}
$$

Alors pour $\left(x_{0}, t_{0}\right)$ fixé, on a

$$
\begin{aligned}
|u|_{m, \Lambda\left(x_{0}, t_{0}\right)} \leq & C_{m} n^{m^{\prime}}\left\{|A(n) u|_{m^{\prime}, \Lambda\left(x_{0}, t_{0}\right)}+|B(n) u|_{m^{\prime}, G\left(x_{0}, t_{0}\right)}\right. \\
& \left.+|u|_{m^{\prime}, D\left(x_{0}, t_{0}\right)}\right\}, \quad \forall u \in C^{\infty}\left(\overline{\boldsymbol{R}_{+}^{3}} \times[0, \infty)\right) .
\end{aligned}
$$

En effet, l'estimation (9.1) en prenant $(x, t)=\left(\frac{x_{0}}{n}, \frac{t_{0}}{n}\right)$ résulte (9.2) d'après le changement des variables $x^{\prime}=n x, t^{\prime}=n t$.

Soit $c\left(s_{0}\right)=c \geq 1$. Prenons 


$$
\begin{aligned}
& \eta_{1}=0, \eta_{2}=\sqrt{c^{2}-1}, p=1, \xi=-c \\
& \psi(x, t)=\left(\xi x_{3}+i \eta_{2} x_{2}+t\right)
\end{aligned}
$$

et

$$
u_{n}^{N}(x, t)=\sum_{j=1}^{N} \exp (n \psi(x, t)) \cdot n^{-j} w_{j}(x, t) .
$$

Par les développement de Taylor des coefficients de $A(n)$ et de $B(n)$ nous avons

$$
\begin{aligned}
& A(n)=n^{2} \cdot \sum_{j=0}^{N} A_{j}\left(x, \frac{\partial}{\partial x}, \frac{\partial}{\partial t}\right) n^{-j}+\widetilde{L}_{N}\left(\frac{x}{n}, \frac{\partial}{\partial x}, \frac{\partial}{\partial t}\right) n^{-N+2} \\
& B(n)=n \cdot \sum_{j=0}^{N} B_{j}\left(x, \frac{\partial}{\partial x}, \frac{\partial}{\partial t}\right) n^{-j}+\widetilde{B}_{N}\left(\frac{x}{n}, \frac{\partial}{\partial x}, \frac{\partial}{\partial t}\right) n^{-N+1} .
\end{aligned}
$$

Notons que $A_{0}=\square$.

Et sans géner la généralité on peut supposer que $B$ s'exprime sous la forme

$$
B_{0}=\frac{\partial}{\partial x_{3}}+b \frac{\partial}{\partial x_{1}}+c \frac{\partial}{\partial t} .
$$

$A_{j}$ sont des opérateurs différentiels d'ordre 2 à coefficients polynome et $B_{j}$ d'ordre 1 à coefficients polynome.

$$
A_{0}\left(\exp (n \psi) w_{j}\right)=\exp (n \psi)\left\{2 n\left(\xi \frac{\partial w_{j}}{\partial x_{3}}+i \eta_{2} \frac{\partial w_{j}}{\partial x_{2}}+p \frac{\partial w_{j}}{\partial t}\right)+\square w_{j}\right\}
$$

et pour $l \geq 1$

$$
A_{l}\left(\exp (n \psi) w_{j}\right)=\exp (n \psi)\left\{n^{2} A_{l 0}\left(w_{j}\right)+n A_{l 1}\left(w_{j}\right)+A_{l 2}\left(w_{j}\right)\right\}
$$

où $A_{l m}, m=0,1,2$ sont des opérateurs différentiels à coefficients polynome d'ordre $m$. Posons

$$
K=2\left(\xi \frac{\partial}{\partial x_{3}}+i \eta_{2} \frac{\partial}{\partial x_{2}}+p \frac{\partial}{\partial t}\right)+A_{10}
$$

Alors

$$
\begin{gathered}
A(n)\left(u_{n}^{N}\right)=\exp (n \psi) \cdot n^{2}\left[n K w_{0}+\left(K w_{1}+A_{20} w_{0}+A_{11} w_{0}\right)\right. \\
+n^{-1}\left\{K w_{2}+A_{20} w_{1}+A_{11} w_{1}+A_{30} w_{0}+A_{21} w_{0}+A_{12} w_{0}\right\} \\
+\cdots+n^{-j}\left\{K w_{j+1}+\left(A_{20}+A_{11}\right) w_{j}+\cdots\right.
\end{gathered}
$$




$$
\left.\left.+\left(A_{j+20}+A_{j+11}+A_{j 2}\right) w_{0}\right\}+\cdots\right]+n^{-N} \tilde{A}_{N}\left(u_{n}^{N}\right) .
$$

Par la même considération on a

$$
\begin{gathered}
B(n) u_{n}^{N}=\exp (n \psi)\left[\left(B_{0} w_{0}+B_{10} w_{0}\right)+n^{-1}\left\{\left(B_{0} w_{1}+B_{10} w_{1}\right)\right.\right. \\
\left.\left.+\left(B_{20} w_{0}+B_{11} w_{0}\right)\right\}+\cdots\right]+\widetilde{B}_{N} u_{n}^{N}
\end{gathered}
$$

Donc nous cherchons $v_{j}$ de telle façon que

$$
\begin{aligned}
& {\left[B_{0} w_{0}+B_{10} w_{0}\right]_{x_{3}=0}=0,} \\
& K w_{0}=0
\end{aligned}
$$

et que pour $j \geq 0$

$$
\begin{aligned}
& K w_{j+1}=-\left\{A_{20} w_{j}+A_{11} w_{j}+\cdots+\left(A_{j+20}+A_{j+11}+A_{j 2}\right) w_{0}\right\} \\
& B_{0} w_{j+1}+B_{10} w_{j+1}=-\left\{\left(B_{20}+B_{11}\right) w_{j}+\cdots+\left(B_{j+20}+B_{j+11}\right) w_{0}\right\} .
\end{aligned}
$$

La substitution de la relation

$$
\left[\frac{\partial w_{0}}{\partial x_{3}}\right]_{x_{3}=0}=\left[-b \frac{\partial w_{0}}{\partial x}-c \frac{\partial w_{0}}{\partial t}-b_{10}(x, t) w_{0}\right]_{x_{3}=0}
$$

qui se déduit de $\left(B_{0}+B_{10}\right) w_{0} !_{x_{3}=0}=0$, à $K w_{0}=0$ nous donne

$$
2(-\xi c+p) \frac{\partial \widetilde{w}}{\partial t}-2 \xi b \frac{\partial \widetilde{w}}{\partial x_{1}}+2 i \eta_{2} \frac{\partial \widetilde{w}}{\partial x_{2}}-\xi b_{10} \widetilde{w}+A_{10} \widetilde{w}=0
$$

où $\widetilde{w}=\left.w_{0}(x, t)\right|_{x_{3}=0}$. Le théorème de Cauchy-Kowalevski permit de l'existence de $\widetilde{w}\left(x_{1}, x_{2}, t\right)$ vérifiant (9.5) dans un voisinage de l'origine et $\widetilde{w}\left(x_{1}, x_{2}, 0\right)=1$, puisque $-\xi c+p \neq 0$. En utilisant encore le théorème de Cauchy-Kowalevski nous avons $v_{0}(x, t)$ satisfaisant à

$$
\left\{\begin{array}{l}
K w_{0}=0 \\
\left.w_{0}\right|_{x_{3}=0}=\widetilde{w}\left(x_{1}, x_{2}, t\right)
\end{array}\right.
$$

dans un voisinage de l'origine. D'après la relation (9.5) que $\widetilde{w}$ vérifie on voit que la fonction $w_{0}$ vérifie (9.3).

Par la même processus on peut trouver $w_{j}(x, t), j=1,2, \cdots, N$ vérifiant (9.4). Puisque $w_{0}(0,0)=1$ on trouve $t_{N}>0, x_{1 N}, x_{2 N}, c_{N}>0$ tels que

$$
\begin{aligned}
& \left|u_{n}^{N}\left(x_{N}, t_{N}\right)\right| \geq c_{N} \cdot \exp \left(n t_{N}\right) \\
& \left|A(n) u_{n}^{N}\right|_{m^{\prime}, \Lambda\left(x_{N}, t_{N}\right)} \leq C_{N, m} \cdot n^{-N+m^{\prime}} \cdot \exp \left(n t_{N}\right)
\end{aligned}
$$




$$
\begin{aligned}
& \left|B(n) u_{n}^{N}\right|_{m^{\prime}, G\left(x_{N}, t_{N)}\right.} \leq C_{N, m} n^{-N+m^{\prime}} \cdot \exp \left(n t_{N}\right) \\
& \left|u_{n}^{N}\right|_{m^{\prime}, D\left(x_{N}, t_{N}\right)} \leq C_{N, m} n^{m^{\prime}},
\end{aligned}
$$

pour tout $n \geq 0$, où $x_{N}=\left(x_{1 N}, x_{2 N}, 0\right)$.

En choisissant $N$ suffisamment grand par rapport à $m^{\prime}$, qui est déterminé par $m=0$, la substitution des estimations au-dessus résulte

$$
c_{N} \cdot \exp \left(n t_{N}\right) \leq C_{N, m} \cdot n^{-N+m^{\prime}} \exp \left(n t_{N}\right)+C n^{m^{\prime}}, \quad \forall n \geq 1 .
$$

C'est une contradiction.

C'est ainsi que nous avons montré la nécessite de la condition (1.3).

Et puis nous allons démontrer que, lorsque la condition (1.3) a lieu, la vitesse maximume est au moins (1.4). Il suffit de considérer le cas où

$$
\sup _{s \in \Gamma} \frac{1+v^{2}}{-c v+\sqrt{1+\left|v^{2}-c^{2}\right|}}=\frac{1+v\left(s_{0}\right)^{2}}{-c\left(s_{0}\right) v\left(s_{0}\right)+\sqrt{1+v\left(s_{0}\right)^{2}-c\left(s_{0}\right)^{2}}}>1
$$

puisque le résultat de Miyatake [9] montre que la vitesse est égale à 1 si $v(s) \leq-c(s)$ sur $\Gamma$. Supposns que

$$
\widetilde{v}_{0}<\frac{1+v\left(s_{0}\right)^{2}}{-c\left(s_{0}\right) v\left(s_{0}\right)+\sqrt{1+v\left(s_{0}\right)^{2}-c\left(s_{0}\right)^{2}}}
$$

où $\widetilde{v}_{0}$ désigne la vitesse maximume du problème $(\mathrm{P})$.

Après la transformation $\Psi$ nous pouvons trouver une constante $\varphi$ vérifiant

$$
v_{0}^{-1}>\varphi>\frac{-c\left(s_{0}\right) v\left(s_{0}\right)+\sqrt{1+v\left(s_{0}\right)^{2}-c\left(s_{0}\right)^{2}}}{1+v\left(s_{0}\right)^{2}}
$$

où $v_{0}$ désigne la vitesse maximume du problème pour $A, \widetilde{B}$ dans un voisinage de l'origine. Alors l'inégarité droite de (9.7) permit de trouver des constante réelles $\eta_{2}>0, \xi<0$ telles que

$$
\begin{aligned}
& \left(1-\varphi^{2}\right)-\left(\xi^{2}+\left(i \eta_{2}\right)^{2}\right)=0 \\
& \xi+(c-b \varphi)=0, \text { où } b=v\left(s_{0}\right) \text { et } c=c\left(s_{0}\right) .
\end{aligned}
$$

Posons

$$
\phi(x, t)=\xi x_{3}+i \eta_{2} x_{2}+\left(t-\varphi x_{1}\right)
$$

Grâce à 


$$
\left(\nabla_{x} \psi\right)^{2}=\psi_{t}^{2}
$$

et à

$$
B_{0} \psi=0
$$

nous pouvons construire une fonction $u_{n}^{N}$ sous la forme

$$
u_{n}^{N}=\exp (n \psi) \sum_{j=0}^{N} w_{j}(x, t) n^{-j}
$$

qui est définie dans $U_{N} \times\left[-t_{N}, t_{N}\right]$ où $U_{N}$ est un voisinage de l'origine et $t_{N}>0$ et satisfait à

$$
\begin{aligned}
& \left|u_{n}^{N}\right| \geq c_{N} \cdot \exp \left(n\left(t-\varphi x_{1}+\xi x_{3}\right)\right) . \quad \forall(x, t) \in \ell_{N} \times\left[-t_{N}, t_{N}\right] \\
& \left|D^{\alpha} A(n) u_{n}^{N}\right| \leq \exp \left(n\left(t-\varphi x_{1}+\xi x_{3}\right)\right) \cdot C_{N, \alpha} n^{-N+|\alpha|+2}, \\
& \forall(x, t) \in U_{N} \times\left[-t_{N}, t_{N}\right] \\
& \left|D^{\alpha} B(n) u_{n}^{N}\right| \leq \exp \left(n\left(t-\varphi x_{1}\right)\right) \cdot C_{N, a} n^{-N+|\alpha|+1}, \\
& \forall(x, t) \in U_{N} \cap\left\{x_{3}=0\right\} \times\left[-t_{N}, t_{N}\right] .
\end{aligned}
$$

Prenons $x_{10}, t_{0}>0$ de façon que

$$
\begin{aligned}
& \left(x_{0}, t_{0}\right)=\left(x_{10}, 0,0, t_{0}\right) \in \mathcal{L}_{N} \times\left[0, t_{N}\right] \\
& v_{0} t_{0}<x_{10}<\frac{1}{\varphi} t_{0} .
\end{aligned}
$$

Pour tout $\left(x_{1}, x_{2}, x_{3}, t\right) \in \Lambda\left(x_{0}, t_{0}\right)$ il a lieu d'après (9. 7)

$$
t-\varphi x_{1}+\xi x_{3} \leq t_{0}-\varphi x_{10} .
$$

Donc nous avons

$$
\begin{gathered}
\left|A(n) u_{n}^{N}\right|_{m^{\prime}, A\left(x_{0}, t_{0}\right)} \leq \exp \left(n\left(t_{0}-\varphi x_{10}\right)\right) \cdot C_{N, m^{\prime}} n^{-N+m^{\prime}+2} \\
\left|B(n) u_{n}^{N}\right|_{m^{\prime}, G\left(x_{0}, t_{0}\right)} \leq \exp \left(n\left(t_{0}-\varphi x_{10}\right)\right) \cdot C_{N, m^{\prime}} n^{-N+m^{\prime}+1} \\
\left|u_{n}^{N}\right|_{m^{\prime}, D\left(x_{0}, t_{0}\right)} \leq C_{N} \cdot n^{m n^{\prime}+N} .
\end{gathered}
$$

D'autre part

$$
\mid u_{n}^{N}\left(x_{0}, t_{0}\right) ! \geq c_{N} \cdot \exp \left(n\left(t_{0}-\varphi x_{10}\right)\right) .
$$

La substitution des estimations au-dessus à (9.2) donne une contradiction si l'on prend $N$ assez grand. Ainsi nous avons démontré que la vitesse maximume de (P) est au moins (1.4). Mais nous arons déjà montré 
dans $\S 2$ que la vitesse maximume est majorée par (1.4). Donc la vitesse maximume est égale à (1.4).

\section{Références}

[1] Erdélyi, A., Asymptotic expansions, Dover Publ., New York, 1965.

[2] Ikawa, M., Mixed problem for the wave equation with an oblique derivative boundary condition, Osaka J. Math., 7 (1970), 495-525.

[3] Ikawa, M., Sur les problèmes mixtes pour l'équation des ondes, Publ. RIMS, Kyoto Univ., 10 (1975), 669-690.

[4] Ikawa, M., Problèmes mixtes pour l'équation des ondes, Publ. RIMS, Kyoto Univ., 12 (1975), 55-122.

[5] Kajitani, K., A necessary condition for the well posed hyperbolic mixed problem with variable coefficients, J. Math. Kyoto Univ., 14 (1974), 231-242.

[6] Kumano-go, H., Opérateurs Pseudo-différentiels (en japonais), Iwanami Shoten, Tokyo, 1975.

[7] Ludwig, D., Uniform asymptotic expansion of the field scattered by a convex object at high frequencies, Comm. Pure Appl. Math., 20 (1967), 103-138.

[8] Miller, J. C. P., Airy integral, Cambridge, 1946.

[9] Miyatake, S., Mixed problem for hyperbolic equation of second order, J. Math. Kyoto Univ., 13 (1973), 435-487.

[10] Mizohata, S., On evolution equations with finite propagation speed, Israel J. Math., 13 (1972), 173-187.

[11] Sakamoto, R., Mixed problems for hyperbolic equations I, J. Math. Kyoto Univ., 10 (1970), 349-373. 